\title{
Decaimento exponencial da função de dois pontos para o Modelo de Ising com acoplamentos aleatórios
}

\author{
José de França Bueno
}

DISSERTAÇÃO APRESENTADA $\mathrm{AO}$ INSTITUTO DE MATEMÁTICA E ESTATÍSTICA DA

UNIVERSIDADE DE SÃO PAULO

PARA OBTENÇÃO DO GRAU DE MESTRE EM MATEMÁTICA APLICADA

\section{Área de Concentração: Mecânica Estatística Orientador: Prof $\stackrel{\circ}{\mathrm{Dr}} \stackrel{\circ}{\circ}$ Henrique von Dreifus}

Durante a elaboração deste trabalho, o autor recebeu apoio financeiro da CAPES 


\section{Decaimento exponencial da função de dois pontos para o Modelo de Ising com Acoplamentos aleatórios.}

Este exemplar corresponde à redação final da dissertação apresentada por José de França Bueno, devidamente corrigida e aprovada pela comissão julgadora.

São Paulo, dezembro de 1996.

Banca examinadora:

Profo Dr o Henrique von Dreifus (orientador)

IME-USP

Prof. Dr. Clodoaldo Grotta Ragazzo

IME-USP

Prof. Dr. Domingos H. Marchetti

IF-USP 


\title{
Resumo
}

Este trabalho dedica-se ao estudo da função de dois pontos no Modelo de Ising no caso em que os acoplamentos entre os sítios são v.a.i.i.d.'s. Neste trabalho apresentamos o decaimento exponencial da função de dois pontos tanto para o caso em que os acoplamentos são de alcance finito quanto ro caso em que tais acoplamentos são de alcance infinito com decaimento exponencial. A partir do decaimento exponencial da função de dois pontos com acoplamentos de alcance infinite e decaimento exponencial obtemos a unicidade da medida de Gibbs para este modelo.

\begin{abstract}
This work is dedicated to the study of the two point function in the Ising Model in the case that the interections are i.i.d.r.v.'s. In this work we present the exponential decay of the two point function in the both cases: the interections are finite range and the interections are infinite range with exponential decay. From the exponential decay of the two point function with interections of infinite range and exponential decay we get the unicity of the Gibbs Measure to this Model.
\end{abstract}




\section{Agradecimentos}

Aos colegas, professores e funcionários: amigos que muito ajudaram e incentivaram no decorrer do meu mestrado.

Ao orientador Henrique: pela dedicação, confiança e infinita paciência demonstrados ao longo deste trabalho. 


\section{Conteúdo}

1 Introdução $\quad 3$

1.1 Modelos Ferromagnéticos . . . . . . . . . . . . . . . . . . 3

2 Desigualdades de Correlação e Aspectos Qualitativos 9

2.1 Desigualdades de Correlação . . . . . . . . . . . . . . . . . . . 9

2.2 Aspectos qualitativos do Modelo de Ising sem desordem . . . . . . . . . . . 11

3 Modelos tipo Ising com Acoplamentos de alcance finito 18

3.1 Acoplamentos de alcance finito e não-aleatórios . . . . . . . . . . . . 18

3.2 Argumento de Peierls . . . . . . . . . . . . . . . . . . . 21

3.3 Acoplamentos de alcance finito e aleatórios . . . . . . . . . . . . . . 24

3.4 Singularidades de Griffiths . . . . . . . . . . . . . . . . . 27

4 Acoplamentos aleatórios de alcance infinito 32

4.1 Decaimento da função de dois pontos . . . . . . . . . . . . . . . . 32

4.2 Independência da Magnetização em relação às condições de Fronteira . . . 38

$\begin{array}{ll}\text { Apêndice } & 42\end{array}$

A Desigualdades de Correlação $\quad 42$

A.1 Primeira Desigualdade de Griffiths . . . . . . . . . . . . . . . . . 42 
A.2 Segunda Desigualdade de Griffiths . . . . . . . . . . . . . . . 43

A.3 Aplicaçòes de G I e G II . . . . . . . . . . . . . . . . . . . . . . . . 44

A.4 Desigualdade FKC . . . . . . . . . . . . . . . . . . . . 45

A.5 Teorema de Lieb-Simon . . . . . . . . . . . . . . . . . . . . 4i 


\section{Capítulo 1}

\section{Introdução}

\subsection{Modelos Ferromagnéticos}

Consideremos modelos que descrevem o comportamento macroscópico de materiais ferromagnéticos a partir das interações entre os constituintes individuais do material (em tais modelos os átomos são distribuídos segundo uma estrutura cristalina apresentando momentos magnéticos não-nulos; $\mathrm{Fe}$ e $\mathrm{Ni}$ são exemplos de materiais ferromagnéticos). Como temos constituintes individuais da ordem do número de Avogrado, a utilização de equações dinâmicas para o estudo de tais modelos não é adequada. Dessa forma, uma outra abordagem ( que não através de equações dinâmicas ) se faz necessária.

Para estudarmos o comportamento de um material, deveremos em algum momento realizar medidas em laboratório das variáveis macroscópicas. Estas variáveis macroscópicas também são chamadas de observáveis. Uma questão importante é que o tempo gasto para efetuar uma medida macroscópica é muito grande quando comparado ao tempo (no nível microscópico) gasto pelas partículas para mudarem de configuração. Dessa forma, o valor obtido do observável representa na verdade uma média temporal do observável calculado sobre as configurações.

Antes de prosseguirmos vamos introduzir a seguinte notação: considere o reticulado $Z^{d}$ (com $d \geq 1$ ); vamos indicar por $\Lambda$ um subconjunto finito de $Z^{d}$. A cada sítio $x \in Z^{d}$ associaremos um valor $\sigma_{x} \in 1,-1$. Denotaremos uma dada configuração por $\sigma=\sigma(x)_{x \in Z^{d}}$ e por $\Omega$ o conjunto de todas as configurações $\sigma$. 
Desta forma. dada $F: \Omega \rightarrow R$, o problema volta-se para o estudo de médias temporais do tipo:

$$
F(\sigma):=\lim _{t \rightarrow \infty} \frac{1}{t} \int_{0}^{t} \mathrm{~F}(\sigma(\mathrm{t})) \mathrm{dt}
$$

onde $F(\sigma)$ representa o observável calculado na configuração $\sigma$ e $\sigma(t)$ representa uma dada configuração no instante $\mathrm{t}$

Faz-se agora a hipótese de equilíbrjo: existe uma medida de probabilidade $\mu$ definida em $\Omega$ tal que as médias temporais dos observáveis para tempos suficientemente longos coincida com as médias do observável sobre o espaço de configurações em relação uma medida $\mu$, ou seja:

$$
\int_{\Omega} F(\sigma) d \mu(\sigma)=\lim _{t \rightarrow \infty} \frac{1}{t} \int_{0}^{t} F(\sigma(t)) d t
$$

O objetivo passa a ser estudar as propriedades da medida de probabilidade $\mu$.

Dado que para a determinação da medida $\mu$ precisa-se conhecer propriedades dinâmicas de um sistema com número de componentes da ordem do número de Avogrado, as considerações acima de caráter heurístico não simplificam o estudo do comportamento macroscópico de materiais ferromagnéticos. Entretanto, tais considerações tornam mais plausível um equacionamento probabilístico para o estudo de tais modelos.

Não é objetivo deste trabalho a caracterização da medida $\mu$ pelo limite (1.1). Nós iremos supor que a medida $\mu$ corresponde uma medida obtida segundo os Postulados de Gibbs. Para a situação em que o sistema efetua trocas de calor (mas não de partículas) com o meio exterior, temos pelo Postulado de Gibbs que a medida de probabilidade no espaço de configurações é dada por (em volumes finitos):

$$
d \mu_{\Lambda}(\sigma)=\frac{e^{-\beta H_{\Lambda}(\sigma)} d \sigma_{\Lambda}}{Z_{\Lambda}}
$$

onde:

$\Lambda$ é um subconjunto finito de $Z^{d}$;

$\Omega_{\Lambda} \subset \Omega$ é o conjunto de configurações $\sigma=\sigma_{x}, x \in \Lambda$

$H_{\Lambda}: \Omega \rightarrow R$

$d \sigma_{\Lambda}$ indica a medida produto dada a priori em $\Omega_{\Lambda}$; 
$\beta=\frac{1}{k \cdot T}$ indica a temperatura inversa, onde $k$ é a constante de Boltzmann e $T>0$ é a temperatura;

e $Z_{1}=\int_{\Omega_{1}} \epsilon^{-3 H_{\Lambda}(\sigma)} d \sigma_{.1}$ definida de modo que $\int d \mu_{\Lambda}(\sigma)$ seja igual a 1 é a função de partiçào do sistema.

É importante observarmos que da maneira como foi definida a medida $\mu_{\mathrm{A}}$ do sistema, a probabilidade de ocorrerem configurações com altos valores de energia é baixa.

Daca a medida $\mu_{\Lambda}$ definimos a medida $\mu$ como sendo o limite fraco

$$
\int_{\Omega} F(\sigma) d \mu(\sigma)=\lim _{\Lambda \rightarrow Z^{d}} \int_{\Omega_{\Lambda}} F_{\Lambda}(\sigma) d \mu_{\Lambda}(\sigma)
$$

No caso particular do Modelo de Ising a medida de probabilidade é dada por

$$
d \mu_{\Lambda}(\sigma)=\frac{\epsilon^{-\beta H_{\Lambda}(\sigma)} d \sigma_{\lambda}}{Z_{\Lambda}}
$$

onde a função $H_{\Lambda}(\sigma): \Omega_{\Lambda} \longrightarrow R$ é definida por:

$$
H_{\Lambda}(\sigma)=-\sum_{<x, y>\in \Lambda} \sigma_{x} J_{x y} \sigma_{y}-\Sigma_{x \in \Lambda} h_{x} \sigma_{x}+\text { "condições de fronteira" }
$$

Neste trabalho estaremos supondo que os acoplamentos $J_{x y}$ são sempre do mesmo sinal. No caso em que $J_{x y}>0$ para todo $(x, y) \in Z^{d} \times Z^{d}$ chamamos de modelo de Ising ferromagnético e no caso em que $J_{x y}<0$ para todo $(x, y) \in Z^{d} \times Z^{d}$ chamamos de modelo de Ising antiferromagnético.

Definimos o conjunto fronteira $(\partial \Lambda)$ de um volume $\Lambda$ como sendo o conjunto de todos os sítios $x \notin \Lambda$. Dizemos que temos fixadas condições de fronteira se, para todo $x \notin \Lambda$, $\sigma_{x}$ possuir algum valor fixado. Além disso, as chamadas condições de fronteira livres correspondem a condição de $J_{x y}=0$ sempre que $x \in \Lambda$ e $y \notin \Lambda$ ou vice-versa.

A partir da função de partição definimos a função Energia Livre:

$$
f(\beta, h)=\lim _{\Lambda \rightarrow Z^{d}} f_{\Lambda}(\beta, h)=-\lim _{\Lambda \rightarrow Z^{d}} \frac{\log \left(Z_{\Lambda}\right)}{\beta|\Lambda|}
$$

( onde chamamos o limite $\Lambda \longrightarrow Z^{d}$ de limite termodinâmico do sistema ) e a partir da função Energia Livre definimos a seguintes funções :

a função magnetização (para volumes finitos):

$$
m_{\Lambda}(\beta, h)=\frac{\partial f_{\Lambda}(\beta, h)}{\partial h}=\frac{1}{|\Lambda|} \sum_{i}<\sigma_{i}>
$$


(onde $<\sigma_{i}>=\frac{1}{\Lambda} \int \sigma d \mu(\sigma)$ )

Para o limite termodinâmico temos:

$$
m(3 . h)=\lim _{1 \rightarrow Z^{d}} \frac{1}{|.1|} m_{\Lambda}(3 . h)
$$

Nos pontos $(\beta, h)$ em que a função Energia Livre é diferenciável temos $m(\beta, h)=$ $-\frac{\partial f(\beta, h)}{\partial h}$.

Nos pontos $(\beta, h)$ em que a função Energia Livre é diferenciável definimos ainda:

a função suscetibilidade magnética:

$$
\chi(\beta, h)=\frac{\partial M(\beta, h)}{\partial h}=-\frac{\partial^{2} f(\beta, h)}{\partial h^{2}}
$$

a função calor específico:

$$
c_{H}(\beta, h)=\frac{\partial^{2} f(\beta, h)}{\partial \beta^{2}}
$$

a função energia interna:

$$
U(\beta, h)=\frac{\partial f(\beta, h)}{\partial \beta}
$$

Um fenômeno que se observa em materiais ferromagnéticos é o fenômeno de transição de fase: no caso do modelo de Ising ferromagnético tal fenômeno se caracteriza pela existência de uma temperatura crítica ( que denotaremos por $T_{c}$ ) tal que: para $T>T_{c}$, a função magnetização $m(\beta, h)$ é analítica em h e $m_{0}(\beta)=\lim _{h \rightarrow 0^{+}} m(\beta, h)=\lim _{h \rightarrow 0^{-}} m(\beta, h)=$ 0 ; no entanto, se pusermos o mesmo material ferromagnético a baixas temperaturas $\left(T<T_{c}\right)$, sob a influência de um campo externo $\mathrm{h}$, a função magnetização deixa de ser analítica em $h$ e teremos $\lim _{h \rightarrow 0^{+}} m(\beta, h)=-\lim _{h \rightarrow 0^{-}} m(\beta, h) \neq 0$. (Numa abordagem heurística dizemos que o material ferromagnético tem uma "memória" da direção dada pelo campo externo quando $T<T_{c}$ ). Concomitantemente ao fenômeno de transição de fase muitas vezes ocorre o fenômeno de quebra de simetria. No caso do Modelo de Ising ( para $h=0$ e sob condições de contorno livres ) a medida $\mu_{\Lambda}(\sigma)$ é invariante sob a transformação $\sigma_{x} \longrightarrow-\sigma_{x}$. Temos também que a função energia livre não depende das condições de fronteira utilizadas para definir o limite termodinâmico. No entanto para $\beta>\beta_{c}$ e dimensão $\geq 2$ existem pelo menos duas fases puras. Chamamos de fase "+" a fase caracterizada pelo fato de que $m_{+}(\beta, h)>0$ e de fase "-" a fase caracterizada pelo fato de que $m_{-}(\beta, h)<0$ o que indica que houve uma quebra de simetria (onde $m_{+}(\beta, h)$ denota a função magnetização com condições de fronteira "+ " e $m_{-}(\beta, h)$ denota a função 
magnetizaçào com condiçòes de fronteira "-.". No caso do modelo de Ising em 2 dimensòes a transição de fase ocorre na presença da quebra de simetria. No entanto. há casos ( por exemplo, na transiçào líquido-gás ) em que a transiçào de fase ocorre na ausência de quebra de simetria.

A transiçào de fase é caracterizada do ponto de vista termodinâmico pela existência de singularidades da funçào de estado Energia Livre. É imediato que para volumes finitos a função Energia Livre é uma função analítica em h. Assim, para caracterizarmos a transição de fase é necessário tcmarmos o limite termodinâmico. L'm resuntado importante no caso de campos externos uniformes é o Teorema de Lee-Yang: no modelo de Ising ferromagnético há uma única fase para $h \neq 0$,ou seja, pontos de não-analiticidade podem ocorrer apenas quando o campo externo h for nulo. Também é possivel considerarmos Modelos tipo Ising em que os acoplamentos $J_{x y}$ são variáveis aleatórias independentes identicamente distribuídas (v.a.i.i.d.). Fisicamente, estes acoplamentos aleatórios estariam modelando as impurezas do material. Tais modelos são chamados também de modelos desordenados. Já observamos que no caso do Modelo de Ising com acoplamentos de primeiros vizinhos determinísticos ocorre o fenômeno de transição de fase ( para d $\geq 2$ ). Observamos também que tal fenômeno está associado com a perda de analiticidade da função Energia Livre $\left(\lim _{h \rightarrow 0^{+}} m(\beta, h) \neq \lim _{h \rightarrow 0^{-}} m(\beta, h)\right)$. O que vamos ver no capítulo 3 é que, para o caso do Modelo de Ising com acoplamentos diluidos ( ou seja: $J_{i j}=1$ com probabilidade $p>0$ ou $J_{i j}=0$ com probabilidade $1-p$ se $|i-j|=1$ e $J_{i j}=0$ se $|i-j|>1$ ) tais singularidades (as quais são chamadas de Singularidades de Griffiths) da função energia livre surgem, mesmo para temperaturas $\mathrm{T}$ para as quais $\lim _{h \rightarrow 0^{+}} m(\beta, h)=\lim _{h \rightarrow 0^{-}} m(\beta, h)$. Observação: a menos de menção em contrário, a notação $|i-j|$ representa a norma euclidiana.

O objetivo deste trabalho é apresentarmos os seguintes resultados: para o modelo de Ising ferromagnético com acoplamentos v.a.i.i.d.'s de alcance infinito e decaimento exponencial, obtemos o decaimento exponencial da função de dois pontos (este resultado indica o caráter local deste tipo de acoplamentos: sua influência não se faz sentir a grandes distâncias). A partir desta informação obteremos o limite termodinâmico e a unicidade da medida de Gibbs para este modelo (quando $\beta<\beta_{c}$ ). Nesta demonstração utilizamos a técnica de duplicação de variáveis.

A estrutura do trabalho é a seguinte: no capítulo 2 fazemos várias considerações de natureza qualitativa do modelo de Ising ferromagnético com acoplamentos de alcance finito; no capítulo 3 apresentamos alguns resultados relacionados com o decaimento da função de dois pontos com acoplamentos de alcance finito (mas de modo que não nos restrinjamos 
ao caso de primeiros vizinhos), no caso em que os acoplamentos sào aleatórios e no caso em que os acoplamentos nào são aleatórios: no capítulo 4 aprensentamos a demonstração do decaimento exponencial da funçào de dois pontos no caso de acoplamentos va.i.i.d. com decaimento exponencial com respeito a distância entre os sítios. Neste capítulo também demonstramos a existência do limite termodinâmico no caso de acoplamentos aleatórios com decaimento exponencial. Este capítulo 4 constitui o núcleo do trabalho. Por último, temos um apêndice onde apresentamos a demonstração de várias desigualdades de correlação. 


\section{Capítulo 2}

\section{Desigualdades de Correlação e Aspectos Qualitativos}

Neste capítulo t,rataremos de desigualdades de correlação, aspectos qualitativos do Modelo de Ising sem desordem para $d \geq 1$ além de uma breve discussão sobre expoentes críticos e relações de escala.

\subsection{Desigualdades de Correlação}

Desigualdades de correlação são ferramentas importantes para o estudo destes modelos ferromagnéticos. Tais desigualdades envolvem valores esperados de produtos do tipo $<\prod_{x \in A} \sigma_{x}>\operatorname{com} x \in A \subset \Lambda \subset Z^{d}$. [Geogii] distingue três classes de desigualdades de correlação:

A: Desigualdades relacionadas com a ordem parcial do espaço de configurações.

Esta classe de desigualdades requer uma relação de ordem no espaço de estados e leva em conta a ordem parcial induzida do espaço de configurações $\Omega_{\Lambda}$. A principal representante desta classe é a desigualdade FKG (Fortuin, Kasteleyn e Ginibre, 1971) 
a qual afirma que se $f$ e $g$ forem funções nào-decrescentes definidas em $\Omega_{\Lambda}$ e $\mu$ é uma distribuiçào de Gibbs entào $\langle f(\sigma) g(\sigma)>-<f(\sigma)\rangle<g(\sigma)>\geq 0$. A demonstraçào desta desigualdade lança mào de uma indução sobre os sítios da rede (ver Apêndice). Uima referência interessante sobre vários resultados ligados à desigualdade FKi se encontra em [Graham].

B. Desigualdades relacionadas com a simetria do espaço de estados ou do potencial.

São desigualdades baseadas em propriedades de simetria do espaço de estados e do potencial. As hipóteses essenciais para este tipo de desigualdade sảo os acoplamentos serem ferromagnéticos e a paridade da medida de probabilidade a priori sob a qual estamos trabalhando (como é o caso no modelo de Ising com as medidas de Gibbs). Como exemplo desta classe podemos tomar as desigualdades de Griffiths:

Griffiths I: considere um subconjunto $A \subset \Lambda$ e $\sigma_{A} \equiv \prod_{x \in A} \sigma_{x}$. Então:

$$
<\sigma_{A}>\geq 0
$$

Griffiths II: considere os conjuntos $A \subset \Lambda$ e $B \subset \Lambda$ e $<\sigma_{A} \equiv \prod_{x \omega A} \sigma_{B} \equiv \prod_{x \in B}$. Então $<\sigma_{A} ; \sigma_{B}>=<\sigma_{A} \sigma_{B}>-<\sigma_{A}><\sigma_{B}>\geq 0$

Apesar das hipóteses serem distintas, as desigualdades FKG e G II têm a mesma tese. Um exemplo de aplicação da segunda desigualdade de Griffiths é a demonstração da existência do limite termodinâmico para algumas funções de correlação (ver Apêndice).

C. Desigualdades Geométricas.

Na demonstração dos teoremas das desigualdades desta classe tem papel primordial argumentos geométricos. São consideradas pertencentes a esta classe principalmente as desigualdades derivadas de Representação de funções de Correlação através de expansões em Random Walk. Como exemplos de desigualdades desta classe temos as desigualdades Gaussianas: Se F é um polinômio com coeficientes positivos entào

$$
<\sigma_{x} F(\sigma)>\leq \sum_{y}<\sigma_{x} \sigma_{y}><\frac{\partial F(\sigma)}{\partial \sigma_{y}}>
$$

e a desigualdade de Lieb-Simon (ver Apêndice). Os resultados obtidos a partir deste tipo de desigualdades estào muitas vezes ligados a estimativas de temperatura crítica, expoentes críticos e ao decaimentos exponencial de funções de correlação. Veremos exemplos de 
aplicações da desigualdade de Lieb-Simon nos capítulos 3 e 4 onde esta é utilizada para demonstrar o decaimento exponencial do valor esperado da funçào de dois pontos.

\subsection{Aspectos qualitativos do Modelo de Ising sem desordem}

Inicialmente vamos comentar os aspectos qualitativos do Modelo de Ising ferromagnéticos com acoplamentos constantes e de primeiros vizinhos. Lembramos que no capítulo anterior foi observado que para estudarmos o Modelo de Ising é importante conhecermos os pontos de não-analiticidade da função Energia Livre. Um resultado importante nesse sentido é dado pelo Teorema de Lee-Yang:

Teorema 2.2.1 Seja $Z_{\Lambda}(\beta, h)$ a função de Partição do Modelo de Ising ferromagnético. Então, se $\operatorname{Re}(h) \neq 0$, a Energia Livre $f(\beta, h)=\lim _{\Lambda \rightarrow Z^{d}} f_{\Lambda}(\beta, h)$ onde $f_{\Lambda}(\beta, h)=\frac{\ln \left(Z_{\Lambda}\right)}{\beta \Lambda}$ é uma função analítica de $h$.

Graficamente:

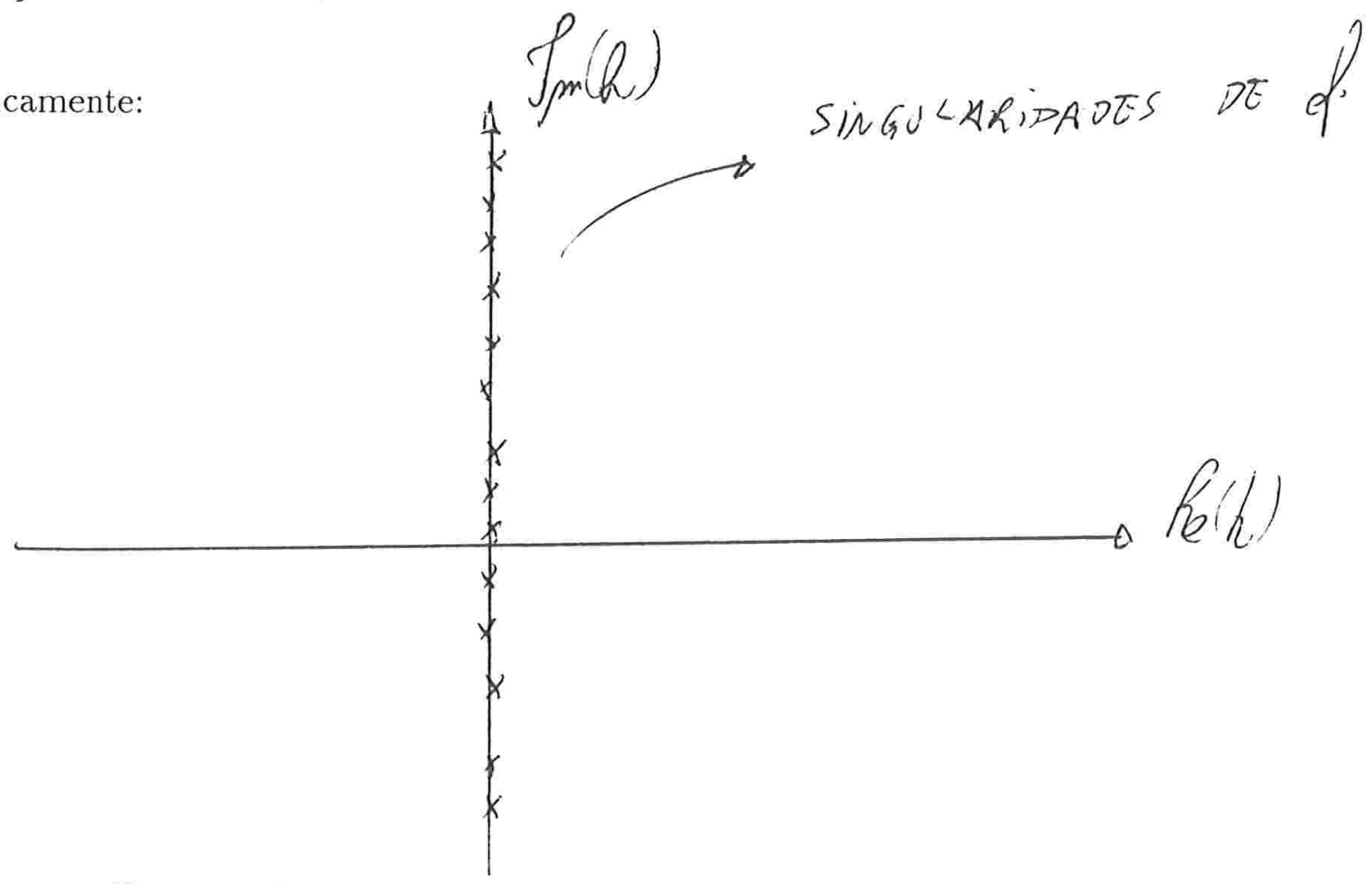

Portanto, o Teorema de Lee-Yang nos diz que para $h \neq 0$, real, não temos transição de fase e a função energia livre pode apresentar singularidades apenas quando $h=0$. Se 
traçarmos o gráfico da magnetizaçào em funçào do campo externo h teremos os gráficos seguintes (fig. 2.2. 2.3. 2.4).
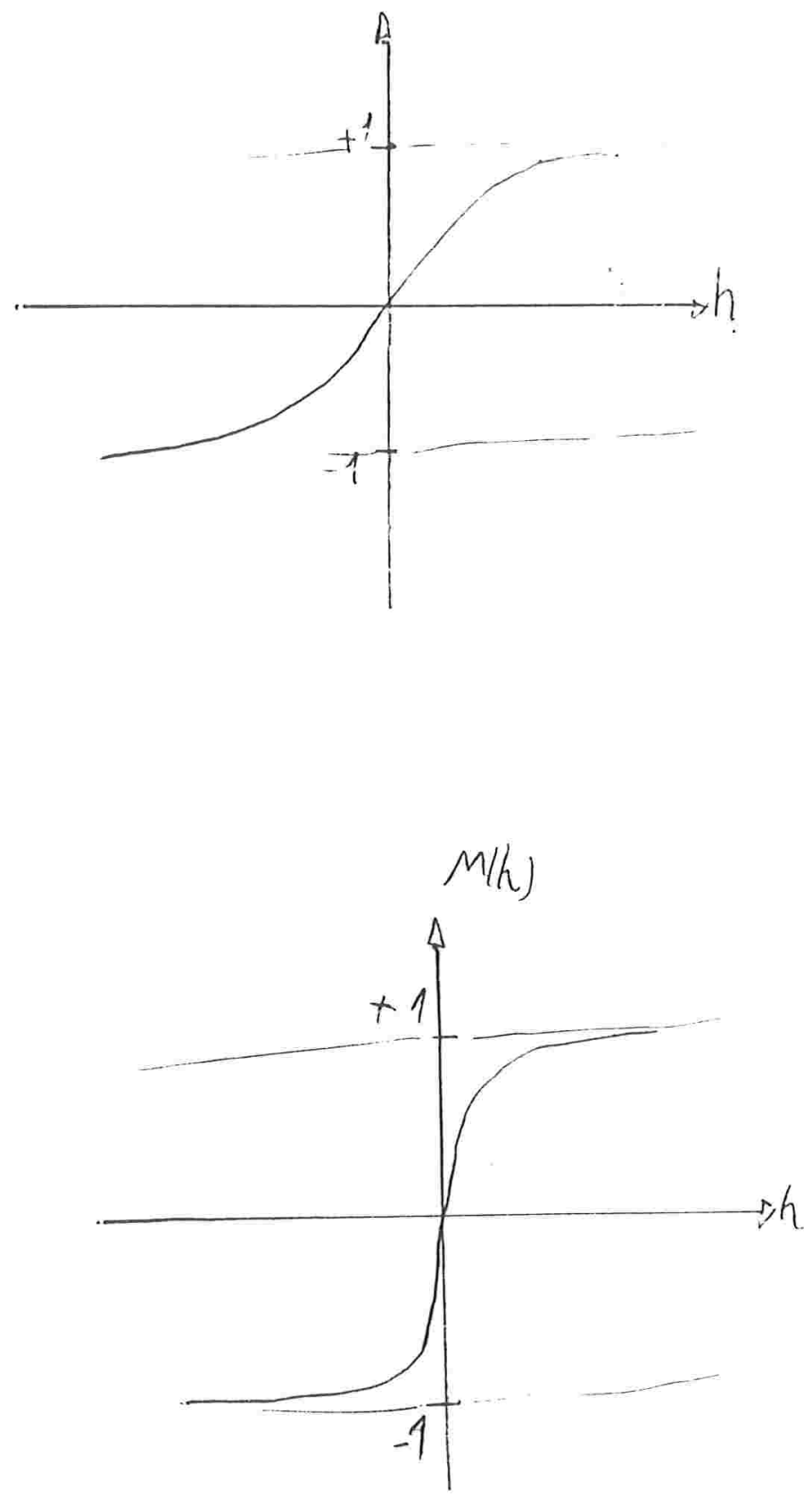


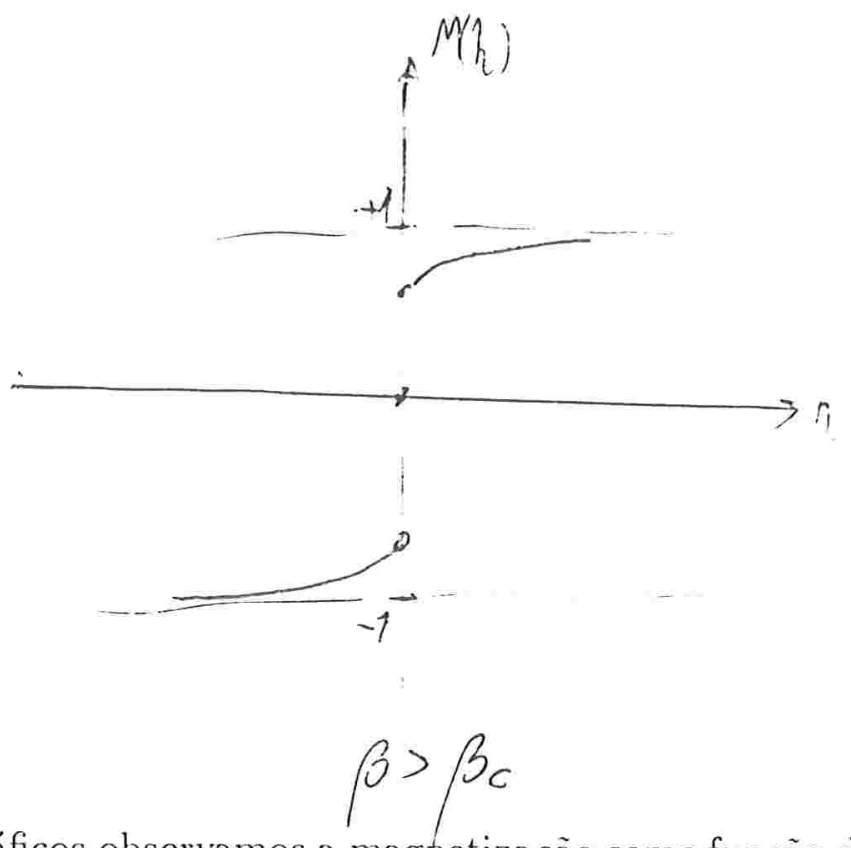

Nestes gráficos observamos a magnetização como função de h para diversos valores de $\beta$. O ponto $(\beta, h)=\left(\beta_{c}, 0\right)$ é chamado de ponto crítico. Observamos que a magnetização é uma função crescente em $h$, e côncava para $h \geq 0$. Observamos ainda que a magnetização possui variações cada vez menores a medida que $h$ aumenta em valor absoluto. A derivada parcial da magnetizaçào em relação a h constitui a funçào suscetibilidade magnética: $\chi(\beta, h)=\frac{\partial m(\beta, h)}{\partial h}$. Apresentamos também os graficos da suscetibilidade magnetica para $\beta<\beta_{c}, \beta=\beta_{c}$ e $\beta>\beta_{c}($ fig 2.5$)$ :

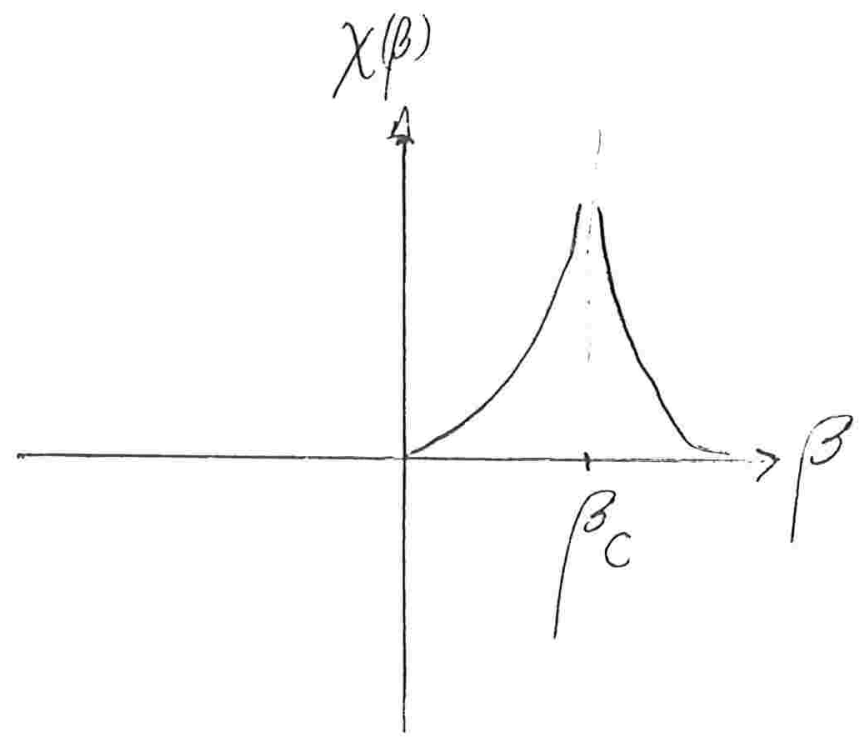


Devido ao fato de as funções de correlação fatorizarem na fase pura, é conveniente introduzir-se as funções de correlação truncadas (ou valor esperado truncado); $\left.\left.<\sigma_{x}: \sigma_{y}\right\rangle=\left\langle\sigma_{x} \sigma_{y}>-<\sigma_{x}\right\rangle<\sigma_{y}\right\rangle$. Para discutirmos aspectos qualitativos deste modelo é importante analisarmos o comportamento do valor esperado $\left\langle\sigma_{x}\right.$; $\left.\sigma_{y}\right\rangle$ como função do campo externo $h$, da temperatura 3 e da distância $|x-y|$. Se $h \neq 0$ já vimos pelo Teorema de Lee-Yang que a função energia livre é analítica. Nesta região não temos transição de fase e as funções de corrleção truncadas decaem exponencialmente. Consideremos o caso $h=0$. Para $\beta=0$ ( temperatura infinita) $\mu_{\Lambda}(\beta, 0)$ reduz-se a medida-produto $\prod_{x \in \Lambda} d \mu_{0}\left(\sigma_{x}\right)$ onde

$\mu_{0}=\frac{1}{2}\left(\delta\left(\sigma_{x}-1\right)+\delta\left(\sigma_{x}+1\right)\right)$. O estado de Gibbs correspondente no limite termodinâmico é a medida produto $\mu=\lim _{\Lambda \rightarrow Z^{d}} \prod_{x \in \Lambda} d \mu_{0}\left(\sigma_{x}\right)$, com respeito a qual os momentos magnéticos são independentes e portanto não estão correlacionados. Para $\beta<\beta_{c}$ as correlações tem um decaimento exponencial (a partir de argumentos de expansão em altas temperaturas) com a distância entre os sítios $|x-y|$ e portanto a função energia livre é analítica como função de $\beta$. O fato das correlações apresentarem um decaimento exponencial com respeito a distância entre os sítios é expresso da seguinte forma:

$$
<\sigma_{x} ; \sigma_{y}>_{\beta, 0} \approx e^{-\frac{|x-y|}{\xi(\beta)}}, \quad \text { para }|x-y| \longrightarrow \infty
$$

Onde $\xi(\beta, 0)<\infty$ é a função comprimento de correlação (ver Ellis, p. 170). Heuristicamente isto significa que as orientações de momentos magnéticos em sítios distantes entre si estão fracamente correlacionadas. A funcao $\xi(\beta, 0)$ é crescente em $\beta$ e as correlações entre os momentos magnéticos se estendem para distâncias cada vez maiores a medida que $\beta$ cresce. Para $\beta \nearrow \beta_{c}$ o comprimento de correlação diverge. Paralelamente, a função suscetibilidade magnética também diverge para $\beta \nearrow \beta_{c}$. O comprimento de correlação infinito está refletido no fato que $\left\langle\sigma_{x} \sigma_{y}\right\rangle_{\beta_{c}}$ não decai mais exponencialmente mas sim decai como uma potência de $|x-y|^{-1}$ :

$$
<\sigma_{x} \sigma_{y}>_{\beta_{c}, 0} \approx|x-y|^{-(d-2+\eta)}, \quad \text { para } \quad|x-y| \longrightarrow \infty
$$

onde d é a dimensão do modelo e $\eta$ é uma constante positiva menor que $1\left(\eta=\frac{1}{4}\right.$ no modelo de Ising em $Z^{2}$ ).

Define-se a magnetização espontânea por

$$
M_{0}(T)=\lim _{h \searrow 0} M(T, h)
$$

onde $\mathrm{T}$ é a temperatura e $\mathrm{M}$ é a função magnetizaçào definida no capítulo anterior. A magnetização espontânea se anula para $T>T_{c}$ e é estritamente positiva para $T<T_{c}$. 
Espera-se (e em alguns casos, como no caso do modelo de Ising para $d=2$ é provado) que $M_{0}(T)$ seja contínua para $T=T_{c}$. seu comportamento qualitativo é dado pela figura:

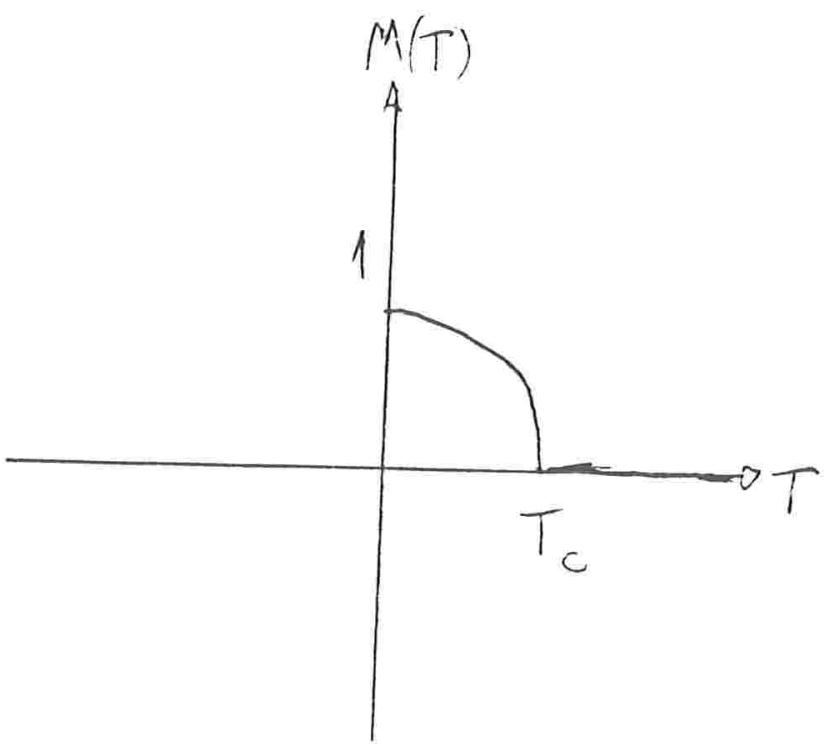

Para $\beta>\beta_{c}$, entramos na região em que o modelo exibe magnetização espontânea. Consideremos a função de correlação de dois pontos com respeito à medida $\mu(\beta, 0)$. Sabemos que (devido à paridade da medida) $\left.\left\langle\sigma_{x}\right\rangle_{\beta, 0,+}=-\left\langle\sigma_{x}\right\rangle_{\beta, 0,-}=m_{+}(\beta, 0)\right\rangle 0$. Como no caso $\beta<\beta_{c}$, a função de correlação truncada decai exponencialmente:

$$
<\sigma_{x} \sigma_{y}>_{\beta, 0,+}-<\sigma_{x}>_{\beta, 0,+}<\sigma_{y}>_{\beta, 0,+}=<\sigma_{x} \sigma_{y}>_{\beta, 0,+}-m_{+}(\beta, 0)^{2} \approx e^{-\frac{|x-y|}{\xi(\beta, 0)}}
$$

para $|x-y| \longrightarrow \infty$ onde $0<\xi(\beta, 0)<\infty$ (para a demonstração da igualdade ver McCoy e Wu; para a demonstração de decaimento exponencial ver Ellis pág. 169). Nesta região ocorre um alinhamento cada vez maior entre os momentos magnéticos.

Ainda : $\lim _{\beta \longrightarrow \infty} m_{+}(\beta, 0)=1$ e $\lim _{\beta \rightarrow \infty} \xi(\beta, 0)=0$.

O comportamento da função suscetibilidade magnética está associado ao do comprimento de correlação para $\beta \rightarrow \beta_{c}$. Temos a seguinte igualdade:

$$
\chi(\beta, h)=\beta \sum_{x \in Z^{d}}<\sigma_{0} ; \sigma_{x}>_{\beta, h}, \quad \text { para } \quad 0<\beta \leq \beta_{c}
$$

Para $\beta \neq \beta_{c} .<\sigma_{0} ; \sigma_{x}>_{\beta, 0}$ decai exponencialmente e portanto $\chi(\beta, 0)$ é finita. No entanto, para $\beta=\beta_{c},<\sigma_{0} ; \sigma_{x}>_{\beta_{c}, 0}$ tem decaimento polinomial da ordem de $|x|^{-\frac{1}{4}} \mathrm{e}$ 
$\backslash\left(\beta_{c}, 0\right)$ é infinita.

Outro aspecto qualitativo destes modelos está relacionado com os chamados expoentes críticos. Estes expoentes críticos estào relacionados com a funçào comprimento de correlação $(\xi(3 . h))$ e com o o comportamento das funçòes termodinâmicas macroscópicas: suscetibilidade magnética $(\backslash(\beta, h))$. e do calor especifico $C_{H}(3 . h)$. Quando nos aproximamos da temperatura crítica $\left(\beta \rightarrow \beta_{c}\right)$ com campo externo nulo $(h=0)$ temos:

$$
\begin{gathered}
\backslash \approx\left|\beta-\beta_{c}\right|^{-\gamma} \\
\xi \approx\left|\beta-\beta_{c}\right|^{-\nu} \\
C_{H} \approx\left|\beta-\beta_{c}\right|^{-\alpha} \\
m \approx\left|\beta-\beta_{c}\right|^{\tilde{\beta}}
\end{gathered}
$$

Um dos fates surpreendentes do modelo é que estes expoentes não são independentes entre si. Há uma relação de dependência entre eles a qual define as chamadas Relações de escala. Tais relações tanto podem ser igualdades entre os expoentes críticos, como por exemplo:

$$
\alpha+2 \tilde{\beta}+\gamma=2
$$

ou

$$
\nu d=2-\alpha
$$

quanto desigualdades entre expoentes críticos. Como exemplo de uma desigualdade entre expoentes críticos temos a desigualdade de Buckingham-Gunton (1969) (ver Ellis p.175) para modelos tipo Ising com acoplamentos não-aleatórios de alcance finito:

$$
2-\eta \leq d \frac{\delta-1}{\delta+1}
$$

onde $\delta$ é o expoente 


$$
m \approx \frac{1}{h^{\delta}}
$$

quando $h \rightarrow 0$ e $3=3$.

Através de argumentos nào rigorosos usando a Teoria de Grupos de Renormalizaçào. acredita-se que estes expoentes críticos não dependam de aspectos locais ou detalhes devidos à particular interaçào mas sim da dimensào da rede onde o modelo está sendo estudado. Quando temos iguaidades e nào a penas desigualdades entre os expoentes críticos chamamos tais relaçōes entre os expoentes críticos de Relaçòes Escala ou de Hiperescala. 


\section{Capítulo 3}

\section{Modelos tipo Ising com Acoplamentos de alcance finito}

Neste capítulo veremos alguns exemplos de modelos tipo Ising em que se demonstra o decaimento da função de correlação quando temos variáveis de acoplamento entre os sítios de alcance finito. Veremos o caso tanto de variáveis determinísticas quanto o caso de variáveis aleatórias i.i.d. Nosso intuito com este capítulo é estudar o que ocorre com este tipo de modelos de modo a termos alguma informação adicional quando, no próximo capítulo, estudarmos modelos com alcance infinito.

\subsection{Acoplamentos de alcance finito e não-aleatórios}

Consideremos o Modelo tipo Ising com interação entre primeiros vizinhos, quando o campo externo h é nulo. Tal modelo é definido pela seguinte Hamiltoniana:

$$
H_{\Lambda}(\sigma)=-\sum_{<x, y>} J_{x y} \sigma_{x} \sigma_{y}
$$


com

$$
J_{x y}= \begin{cases}J_{x y}=J & \text {. se }|x-y|=1 \\ 0 & \text {. caso contrário }\end{cases}
$$

Temos entào o seguinte teorema na versào apresentada por [Dreifus]:

Teorema 3.1.1 : Seja $\Lambda_{L}(x)$ um cubo finito em $Z^{d}$ com lado $L=2 k,(k=1,2,3, \ldots), e$ centro em $x \in Z^{d}$. Se:

$$
\beta J \sum_{\omega \in \partial \Lambda_{L}(x)}<\sigma_{x} \sigma_{\omega}>_{\Lambda_{L}(x)} \leq \delta<1
$$

Então, para $y \in Z^{d}$ com $|x-y| \geq L$ temos:

$$
<\sigma_{x} \sigma_{y}>\leq C e^{-\frac{|x-y|}{\xi(\beta)}}
$$

onde Cé uma constante positiva e

$$
-\xi(\beta)^{-1}=\frac{1}{L} \log (\delta)
$$

\section{Demonstração}

A demonstração deste teorema será feita para o caso de primeiros vizinhos apenas por uma questão de simplicidade. O mesmo resultado vale para acoplamentos de alcance finito com as devidas adaptações na demonstração.

Definimos os seguintes conjuntos:

$$
\begin{aligned}
& \Lambda_{L}(x)=\left\{y \in Z^{d} \operatorname{tq}|x-y|_{\infty} \leq L\right\} \\
& \text { onde }|x-y|_{\infty}=\max _{j=1,2, \ldots, d}\left|x_{j}-y_{j}\right| \\
& \partial \Lambda_{L}(x)=\left\{y \in \Lambda^{c} \operatorname{tq}|z-y|=1, z \in \Lambda_{L}(x)\right\} \\
& \partial^{*} \Lambda_{L}(x)=\left\{y \in \Lambda^{c}:|z-y|=L+1, z \in \Lambda_{L}(x)\right\}
\end{aligned}
$$

Tomando:

$$
X=\left\{(u, v) \in Z^{d} \times Z^{d} \operatorname{tq} \text { ou }\left\{u \in \Lambda_{L}(x) \text { e } v \notin \Lambda_{L}(x)\right\} \text { ou }\left\{u \notin \Lambda_{L}(x) \text { e } v \in \Lambda_{L}(x)\right\}\right\}
$$


Definimos $<()>._{X}$ colocando $J_{u v}=0 \forall u v \in X^{\prime}$ na definiçào de $<()>._{\Lambda}$.

Da desigualdade de Lieb-Simon (ver Apêndice). temos:

$<\sigma_{x} \sigma_{y}>_{\Lambda}-<\sigma_{x} \sigma_{y}>_{X} \leq \sum_{(u, v) \in X}<\sigma_{x} \sigma_{u}>_{X} \beta J<\sigma_{v} \sigma_{y}>_{A}$

Da definiçào do conjunto $X^{\prime}$ sabemos que $<\sigma_{x} \sigma_{y}>_{X}=0$. Assim. a desigualdade acima fica

$<\sigma_{x} \sigma_{y}>_{\Lambda} \leq \sum_{(u, v) \in X}<\sigma_{x} \sigma_{u}>_{X} \beta J<\sigma_{v} \sigma_{y}>_{\Lambda}$

Seja $\bar{s} \in \partial^{*} \Lambda_{L}(x)$ tal que $<\sigma_{\bar{s}} \sigma_{y}>_{\Lambda}=\max _{s \in \tilde{\partial}^{*} \Lambda_{L}(x)}\left\{<\sigma_{s} \sigma_{y}>_{\Lambda}\right\}$ e $\tilde{J}=\max _{(s, t) \in X} J_{s t}$.

Então, $<\sigma_{x} \sigma_{y}>_{\Lambda} \leq\left\{\beta \tilde{J} \sum_{t \in \partial \Lambda_{L}(x)}<\sigma_{x} \sigma_{t}>_{X}\right\}<\sigma_{\bar{s}} \sigma_{y}>_{\Lambda}$

Portanto, se

$$
\beta \tilde{J} \sum_{t \in \partial \Lambda_{L}(x)}<\sigma_{x} \sigma_{t}>_{X} \leq \delta<1
$$

teremos: $<\sigma_{x} \sigma_{y}>_{\Lambda} \leq \delta<\sigma_{\bar{s}_{1}} \sigma_{y}>_{\Lambda} \operatorname{com} \bar{s}_{1} \in \partial^{*} \Lambda_{L}$.

Repetindo o mesmo argumento para $\left\langle\sigma_{\bar{s}_{1}} \sigma_{y}\right\rangle$ com o conjunto

$\Lambda_{L}\left(\bar{s}_{1}\right)=\left\{z \in Z^{d} \operatorname{tq}\left|\bar{s}_{1}-z\right|_{\infty} \leq L\right\}$ obtemos $<\sigma_{x} \sigma_{y}>\leq \delta<\sigma_{s_{1}} \sigma_{y}>\leq \delta^{2}<$ $\sigma_{s_{2}} \sigma_{y}>$

Este argumento pode ser repetido iterativamente desde que $\bar{s}_{k} \notin \Lambda_{L}(y)$ e, desta forma obtemos a quota:

$$
<\sigma_{x} \sigma_{y}>\leq \delta^{\frac{|x-y|}{L+1}}
$$

$\Rightarrow$

$$
<\sigma_{x} \sigma_{y}>\leq e^{-\frac{|x-y|}{\xi(\beta)}}
$$

onde

$$
-\xi(\beta)^{-1}=\frac{1}{L+1} \ln (\delta(\beta))
$$

Um corolário importante desta análise é: 
Corolário 3.1.1 Para $3=3$ c a funçào de dois pontos satisfaz:

$$
<\sigma_{x} \sigma_{y}>\left(3_{c}\right) \geq c t \epsilon|x|^{-\lambda+2-\eta}
$$

$\operatorname{com} \eta \leq d$

Demontração : Inicialmente devemos observar que $\left\langle\sigma_{x} \sigma_{y}\right\rangle\left(\beta_{c}\right)$ não pode ter decaimento exponencial pois estaria em contradição com a própria definição de $\beta_{c}=$ $\sup \left\{\beta:<\sigma_{x} \sigma_{y}>\leq \epsilon^{\frac{-|x-y|}{\xi}}\right.$ quando $|x-y| \rightarrow \infty$, para algum $\left.\xi>0\right\}$. Também não podemos ter $\left\langle\sigma_{x} \sigma_{y}>_{\Lambda} \leq<\sigma_{x} \sigma_{y}>\left(\beta_{c}\right) \leq C|x|^{-d+2-\eta}\right.$ com $\eta \geq d+\varepsilon$ pois isto implicaria que a hipótese do teorema estaria satisfeita e portanto teríamos decaimento exponencial para $<\sigma_{x} \sigma_{y}>\left(\beta_{c}\right)$.

Observação: Se

$$
J_{x y}= \begin{cases}J_{x y}=J, & \text { se }|x-y| \leq N \\ 0, & \text { caso contrário }\end{cases}
$$

$\operatorname{com} N>1$, teremos o mesmo resultado, com as devidas alterações na demınstração.

\subsection{Argumento de Peierls}

Na última seção vimos condições para que tivessemos decaimento exponencial da função de dois pontos. A partir do decaimento exponencial da função de dois pontos podemos demonstrar que não temos transição de fase no sistema (esta demonstração será dada no próximo capítulo). A partir do resultado clássico dado pelo argumento de Peierls (para referência ver Glimm-Jaffe)podemos demonstrar a existência de transição de fase para sistemas em dimensão $d \geq 2$ para o modelo de Ising com acoplamentos positivos nãoaleatórios e de primeiros vizinhos quando estamos a uma temperatura positiva. Apenas por questão de maior simplicidade para expormos a idéia do argumento consideraremos o caso $d=2$ com acoplamentos de primeiros vizinhos. Supomos condições de fronteira " + " (também poderíamos considerar condições de fronteira " - " fazendo as devidas adaptações).

A idéia de Peierls foi associar um conjunto de curvas fechadas chamadas de contornos a cada configuração $\sigma \in \Omega_{\Lambda}$ e, a partir destas curvas efetuar estimativas de entropia e energia para o sistema. Definimos o bordo $\partial \Lambda$ ( ou bd $\Lambda$ ) do volume finito $\Lambda \subset Z^{d}$ como 
sendo o conjunto de todos os sítios $j \in \Lambda^{\circ}$ os quais estào a distância unitária de $\Lambda$. Como as interações $J_{x y}$ sào supostas de primeiros vizinhos, o bordo de 1 contém todos os sítios da rede que interagem com os sítios de .1 . A condição externa " + " fixa a cada spin na fronteira de 1 o valor +1 . Sem perda de generalidade estaremos fixando a condiçào de fronteira " + ". Contornos sào construídos levando-se em consideraçào as eventuais trocas de sinal presentes em uma configuração $\sigma$. Para cada par $(x, y) \in Z^{d},|x-y|=1$, verificamos os sinais de $\sigma_{x}$ e $\sigma_{y}$. Se $\sigma_{x} \neq \sigma_{y}$, traçamos um segmento unitário que bisecta o segmento que une x a y. Dada uma configuração $\sigma$ denotamos por $\Gamma(\sigma)$ o conjunto de segmentos assim construídos. O conjunto de todos os $\Gamma(\cdot)$ é denotado por $\partial \Delta$. A condição externa " + "fixa a cada spin na fronteira de $\Lambda$ o valor +1 e a condição externa " - " fixa a cada spin na fronteira de $\Lambda$ o valor -1 . Dada uma configuração $\sigma$, a hamiltoniana $H_{\Lambda}(\sigma)$ definida por:

$$
H_{\Lambda}(\sigma)=J \sum_{(x, y) \in \Lambda} \sigma_{x} \sigma_{y}-J|\Lambda|
$$

satisfaz: $H_{\Lambda}(\sigma)=2 \beta J|\Gamma(\sigma)|$ onde $|\Gamma(\sigma)|$ é o comprimento do contorno $\Gamma(\sigma)$.

Considerando o caso em que as condições de fronteira são positivas. as componentes $\gamma$ dos contornos são fechadas e estão isoladas da fronteira $\partial \Lambda$. Dada uma componente simples, $\gamma$, definimos o evento $\partial \Delta_{\gamma}$ como: $\partial \Delta_{\gamma}=\{\Gamma(\sigma) \supset \gamma\} \subset \partial \Delta$ isto é, o conjunto de todas as configurações que possuem, na sua representação através de contornos, a componente $\gamma$.

Então a probabilidade do evento $\partial \Delta_{\gamma}$ é denotada por:

$$
\operatorname{Pr}(\gamma)=\frac{\sum_{\Gamma \in \partial \Delta_{\gamma}} e^{-\beta H(\Gamma)}}{\sum_{\Gamma \in \partial \Delta} e^{-\beta H(\Gamma)}}
$$

Teorema 3.2.1

$$
\operatorname{Pr}(\gamma) \leq e^{-2 \beta J|\gamma|}
$$

onde $|\gamma|$ representa o comprimento do contorno $\gamma$.

Demonstração: Vamos representar por $\Gamma_{\gamma}^{*}$ a configuração obtida de $\Gamma_{\gamma}$ trocandose o sinal dos sítios no interior de contorno $\gamma$ (dessa maneira estaremos removendo a componente $\gamma$ do conjunto $\left.\Gamma_{\gamma}\right)$. Então:

$$
\frac{e^{-\beta H\left(\Gamma_{\gamma}\right)}}{e^{-\beta H\left(\Gamma_{\gamma}^{*}\right)}}=e^{-2 \beta J|\gamma|}
$$


Existe uma correspondência biunivoca entre os contornos $\Gamma_{\text {, que contém a compo- }}$ nente $\gamma$ e os contornos que nào contém $\gamma$ obtida através da troca de sinal dos momentos magnéticos dos sítios no interior de $\gamma$. Utilizando esta correspondência vemos que:

$$
\operatorname{Pr}(\gamma)=\frac{\sum_{\Gamma_{\gamma}} e^{-3 H\left(\Gamma_{\gamma}\right)}}{\sum_{\Gamma} e^{-\beta H\left(\Gamma^{\prime}\right)}} \leq \frac{\sum_{\Gamma_{\gamma}} e^{-3 H\left(\Gamma_{\gamma}\right)}}{\sum_{\Gamma_{\gamma}^{*}} e^{-\beta H\left(\Gamma_{\gamma}^{*}\right)}}=\epsilon^{-2 \beta J|\gamma|}
$$

Sendo que a desigualdade segue do fato de termos diminuído o valor da soma no denominador da primeira fração e assim passamos a ter a seguinte desigualdade: $\sum_{\Gamma} e^{-\beta H(\Gamma)} \geq$ $\sum_{\Gamma_{\gamma}^{*}} e^{-\beta H\left(\Gamma_{\gamma}^{*}\right)}$.

Teorema 3.2.2 Para $\beta$ suficientemente grande e $d \geq 2$ temos que:

$$
0 \leq 1-<\sigma_{x}>_{\Lambda,+} \leq e^{-\beta J}
$$

Demonstraçâo: Temos que $1-<\sigma_{x}>_{\Lambda,+}=<1-\sigma_{x}>_{\Lambda,+}$ sendo que apenas configurações com $\sigma_{x}=-1$ contribuem para este valor esperado. Para estas configurações existe necessariamente uma componente $\gamma$ que possui x no seu interior ( dada a condição de fronteira positiva em $\Lambda$ ). Dada uma destas componentes, denotamos por $\partial \Delta_{\gamma}$ o conjunto de todos os contornos $\Gamma$ para os quais a menor componente que contém $x$ em seu interior seja $\gamma$.

Então:

$$
\begin{gathered}
1-<\sigma_{x}>_{\Lambda,+}=2 \frac{\sum_{\gamma} \sum_{\Gamma \in \partial \Delta_{\gamma}} e^{-\beta H(\Gamma)}}{\sum_{\Gamma \in \partial \Delta} e^{-\beta H(\Gamma)}} \\
1-<\sigma_{x}>_{\Lambda,+} \leq 2 \sum_{\gamma} \operatorname{Pr}(\gamma) \leq 2 \sum_{\gamma} e^{-2 \beta J|\gamma|}
\end{gathered}
$$

Seja agora $N(|\gamma|)$ o número de possíveis $\gamma$ 's de comprimento $|\gamma|$ tais que x está no interior de $\gamma$. Este número pode ser estimado a partir dos seguintes fatos:

1 - $\gamma$ está contido em uma região de volume $|\gamma|^{d}$ centrada em x.

2 - começando de qualquer elemento $\gamma$ existem $c=2 d-1$ possibilidades de adicionar um próximo elemento. 
Desta forma $N(|\jmath|) \leq|\gamma|^{d} c^{\mid-1} \mid$. o que implica na seguinte estimativa:

$$
1-<\sigma_{x}>_{1 .+} \leq 2 \sum_{l=4}^{\infty} l^{d} c^{l} c^{-23.3 l}
$$

Tomando $\beta J$ grande segue que:

$$
0 \leq 1-<\sigma_{x}>_{\Lambda,+} \leq \epsilon^{-\beta J}
$$

\subsection{Acoplamentos de alcance finito e aleatórios}

Consideremos o Modelo tipo Ising com interação aleatória entre primeiros vizinhos e com campo externo nulo. Este modelo é definido pela seguinte Hamiltoniana:

$$
H_{\Lambda}(\sigma)=-\sum_{<x, y>} J_{x y} \sigma_{x} \sigma_{y}
$$

onde

$$
J_{x y}= \begin{cases}J_{x y}, \text { se }|x-y| \leq 1 & \\ 0, & \text { caso contrário }\end{cases}
$$

e $J_{x y}$ são v.a.i.i.d.'s.

Teorema 3.3.1 Seja $\Lambda_{L}(x)$ um cubo finito de lado $L=2 k ;(k=1,2,3, \ldots)$, e centro em $x \in Z^{d}$. Se

$$
\beta^{2} n(d)(L+4)^{d-1} \bar{J}^{2} \sum_{w \in \partial \Lambda_{L}(x)} \overline{<\sigma_{x} \sigma_{w}>\Lambda_{L}(x)} \leq \delta<1
$$

onde $n(d)$ denota o número de hiperfaces do cubo de dimensão d.

Então, para $|x-y| \gg L$,

$$
\overline{<\sigma_{x} \sigma_{y}>} \leq C e^{-\frac{|x-y|}{\xi(\beta)}}
$$


onde C'é uma constante positiva $\epsilon$

$$
\begin{aligned}
-\xi(\beta)^{-1} & =\frac{1}{L} \ln (\delta(\beta)) \\
\operatorname{com} \delta(\beta)=(L+4)^{d-1} n(d) \beta^{2} \bar{J}^{2} \sum_{u \in \partial \Lambda_{L}(x)} & {\overline{\left\langle\sigma_{x} \sigma_{y}\right\rangle}}_{\partial \Lambda_{L}(x)}
\end{aligned}
$$

\section{Demonstração}

Definimos os seguintes conjuntos:

$\Lambda_{L}(x)=\left\{z \in Z^{d} \operatorname{tq}|x-z|_{\infty} \leq L\right\}$

$\partial \Lambda_{L}(x)=\left\{z \in \Lambda^{c} \operatorname{tq}|x-z|=1\right\}$

$X=\left\{(u, v) \in Z^{d} \times Z^{d}\right.$ tq ou $\left\{u \in \Lambda_{L}(x)\right.$ e $\left.v \notin \Lambda_{L}(x)\right\}$ ou $\left\{u \notin \Lambda_{L}(x)\right.$ e $\left.\left.v \in \Lambda_{L}(x)\right\}\right\}$

Da desigualdade de Lieb-Simon (ver Apêndice) temos, para $|x-y| \gg L$

$$
<\sigma_{x} \sigma_{y}>\leq \sum_{(t, s) \in \partial \Lambda_{L}(x)}<\sigma_{x} \sigma_{t}>_{\Lambda_{L}(x)} \beta J_{t s}<\sigma_{s} \sigma_{y}>
$$

Aplicando a desigualdade de Lieb-Simon para $\left\langle\sigma_{s} \sigma_{y}>\right.$ com conjunto $\Lambda_{L+1}(x)$, temos:

$$
<\sigma_{s} \sigma_{y}>\leq \sum_{(u, v) \in \partial \Lambda_{L+1}(x)}<\sigma_{s} \sigma_{u}>\beta J_{u v}<\sigma_{v} \sigma_{y}>_{\Lambda_{L+1}(x)}
$$

Usando a limitação dos spins ( $\left|\sigma_{z}\right| \leq 1, \forall z \in Z^{d}$ no caso do modelo de Ising ) temos $\left|<\sigma_{s} \sigma_{u}>\right| \leq 1$.

Portanto:

$$
<\sigma_{x} \sigma_{y}>\leq \sum_{(t, s) \in \partial \Lambda_{L}(x)} \sum_{(u, v) \in \partial \Lambda_{L+1}(x)} \beta^{2} J_{t s} J_{u v}<\sigma_{x} \sigma_{t}>_{\Lambda_{L}(x)}<\sigma_{v} \sigma_{y}>_{\Lambda_{L+1}^{c}}(x)
$$

Notamos agora que, do lado direito da desigualdade acima, $\left\langle\sigma_{x} \sigma_{t}\right\rangle_{X}(J)$ é uma função dos valores $J_{q r}$ com $q, r \in \Lambda_{L}(x)$ e que $\left\langle\sigma_{w} \sigma_{y}>_{X}(J)\right.$ é uma função dos valores $J_{q r}$ com $q, r \notin \Lambda_{L+2}(x)$ temos que a média sobre as configurações $\mathrm{J}$ é fatorizada:

$$
\overline{<\sigma_{x} \sigma_{y}>} \leq \beta^{2} \bar{J}^{2} \sum_{t \in \partial \Lambda_{L}(x)} \sum_{v \in \partial \Lambda_{L+1}(x)} \overline{\left\langle\sigma_{x} \sigma_{t}>_{\Lambda_{L}(x)}\right.} \overline{<\sigma_{v} \sigma_{y}>_{\Lambda_{L+1}^{c}(x)}}
$$


Tomando $w_{0} \in I_{L+2}(x)$ tal que

$$
{\overline{\left\langle\sigma_{u o} \sigma_{y}\right.}}_{\Lambda_{\Lambda_{L+1}^{c}(x)}}=\max _{s \in \Lambda_{L+2}(x)} \overline{\left\langle\sigma_{s} \sigma_{y}\right\rangle_{\Lambda_{L+1}(x)}}
$$

e usando a hipótese do teorema teremos:

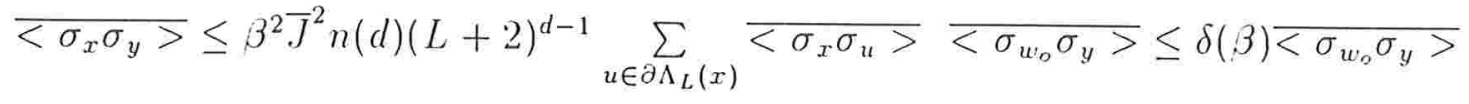

onde $\delta(\beta)=\beta^{2} \bar{J}^{2} n(d)(L+2)^{d-1} \sum_{u \in \partial \Lambda_{L}(x)} \overline{<\sigma_{x} \sigma_{u}>\partial \Lambda_{L}(x)}$

Podemos repetir o mesmo procedimento pelo menos $\frac{|x-y|}{L+2}$ vezes e assim concluímos que:

$$
<\sigma_{x} \sigma_{y}>\leq \delta^{\frac{|x-y|}{L+2}}
$$

e portanto

$$
\overline{<\sigma_{x} \sigma_{y}>} \leq e^{-\frac{|x-y|}{\xi(\beta)}}
$$

com

$$
-\xi(\beta)^{-1}=\frac{1}{L+2} \ln (\delta(\beta))
$$

Corolário 3.3.1 Para $\beta=\beta_{c}$, a função de dois pontos satisfaz:

$$
<\sigma_{x} \sigma_{y}>\left(\beta_{c}\right) \geq c t e|x|^{-d+2-\eta}
$$

com $\eta \leq d$

Demonstração: a demonstração é análoga ao caso não-aleatório.

Observação: Se no lugar de acoplamentos de primeiros vizinhos, tivessemos acoplamentos de alcance finito entre os $\mathrm{N}$ primeiros vizinhos, teríamos o mesmo resultado apenas com algumas modificações na demonstração do teorema e nas constantes. 


\subsection{Singularidades de Griffiths}

Modelos cuja hamiltoniana para um volume finito $\Lambda \subset Z^{d}$ é dada por

$$
H_{\Lambda}(\sigma, J)=-\sum_{(x, y) \in \Lambda} J_{x y} \sigma_{x} \sigma_{y}+h \sum_{x \in \Lambda} \sigma_{x}
$$

onde $J=\left\{J_{x y},(x, y) \in Z^{d}\right\}$ é uma família de variáveis aleatórias i.i.d. satisfazendo $J_{x y} \geq 0(\operatorname{com}|x-y|=1)$ são ditos modelos diluídos. Se $J_{x y}$ são da forma:

$$
J_{x y}= \begin{cases}1 & , \text { com probabilidade } p>0, \text { se }|x-y|=1 \\ 0 & , \text { com probabilidade } 1-p, \text { se }|x-y|=1 \\ 0 & , \text { se }|x-y|>1\end{cases}
$$

dizemos que temos 'diluicão nos acoplamentos' enquanto que se $J_{x y}$ forem da forma:

$$
J_{x y}= \begin{cases}J \tau_{i} \tau_{j} & , \text { para }|i-j|=1 \\ 0 & , \text { caso contrário }\end{cases}
$$

com $\tau_{i}=1$ com probabilidade p e $\tau_{i}=0$ com probabilidade $1-p$ dizemos que temos diluição nos sítios.

Em um artigo de 1969 [Griffiths] mostrou que para modelos com diluição nos sítios a função magnetização deixa de ser uma função analítica em $\mathrm{h}$ para $h=0$ para valores de temperatura abaixo daquela onde ocorre a transição de fase (do caso sem diluição nos sítios.

Os argumentos dados por Griffiths são baseados no teorema de Lee-Yang [ver Ruelle]. Ele estudou o comportamento da magnetização em função de $z=\epsilon^{\beta h}$ e observou que ela é analítica para $|z|<1$ e não pode ser extendida analiticamente através do círculo unitárir Podemos melhor compreender este fenômeno se considerarmos a seguinte situação. sideremos o modelo com sítios diluídos e suponhamos $p<p_{c}(d)$, onde $p_{c}(d)$ é o valor crítico para a probabilidade de ocupação de um sítio no problema de percolação por sítios em $Z^{d}$. Temos, com probabilidade um, nesta situação, apenas "clusters" finitos de sítios acoplados aos seus vizinhos mais próximos de tal maneira que o sistema se decompõe em uma coleção de subsistemas independentes finitos. Neste caso, podemos concluir que com 
probabilidade um, nào há nem magnetizaçào nem long-range order para todos os valores da temperatura. Entretanto. como consequência da lei dos grandes números. também com probabilidade um. existem caixas arbitrariamente grandes dentro das quais todos os sítios estão acoplados. Agora. se $3 . J>\beta_{c}(d)$ ( onde $\xi_{c}(d)$ é o valor crítico para um sistema homogêneo d-dimensional com acoplamentos $J_{x y}=J \forall\langle x . y\rangle \in Z^{d}$ ) teremos caixas arbitrariamente grandes ( mas finitas) dentro das quais o sistema está fortemente correlacionado. A situaçào acima sugere o mecanismo geral para o fenômeno.

Veremos aqui a demonstração destas observaçõos. Esta demonstração está em [Fröhlich].

Antes de demonstrarmos o teorema de Griffiths faremos o cálculo da magnetização. Vamos trabalhar com o modelo de sítios diluídos mas o modelo com diluição nos acoplamentos também pode ser tratado. Diremos que um sítio j está ocupado se e só se $\tau_{j}=1$, caso contrário j será dito vazio. Denotemos por C uma configuração arbitrária de sítios ocupados em $\Lambda$ ( i.e. uma família de aglomerados conexos e disjuntos de sítios ocupados. Além disso, $|C|=$ número de sítios pertencentes a $\mathrm{C}$,

$P_{C, \Lambda}=$ probabilidade de ocorrer $\mathrm{C}$,

$M_{\Lambda}=$ magnetização média por sítio em $\Lambda$

$M_{C}=$ magnetização média por sítio em $\mathrm{C}$.

Então

$$
M_{\Lambda}=|\Lambda|^{-1} \sum_{C \subset \Lambda}|C| P_{C, \Lambda} M_{C}
$$

Seja $z=e^{-2 \beta h}$ e denotemos por $f_{C}$ a energia livre por sítio do sistema restrito a $\mathrm{C}$, i.e.

$$
\beta f_{C}=-|C|^{-1} \log Z_{C}
$$

onde $Z_{C}$ é a função de partição. Então $M_{C}=\frac{\partial f_{C}}{\partial h}=\frac{\partial z}{\partial h} \cdot \frac{\partial f_{C}}{\partial z}=-2 \beta z \frac{\partial f_{C}}{\partial z}$

Escrevendo $e^{-\beta h \sigma_{i}}=e^{\beta h} e^{-\beta h\left(\sigma_{i}+1\right)}$ e somando sobre todos os valores de $\sigma_{i}, i \in C$, vemos que $e^{-\beta h|C|} Z_{C}$ é um polinômio em z de grau $|C|$. Donde

$$
Z_{C:}=\operatorname{cte}^{-|C| / 2} \prod_{\alpha=1}^{|C|}\left(z-\zeta_{\alpha}(C)\right),
$$


onde $\zeta_{\alpha}(C)$ é o $\alpha$-ésimo zero de $Z_{C}$. O Teorema de Lee-Yang diz-nos que

$$
\left|\zeta_{\alpha}(C)\right|=1
$$

para todo $\alpha$. Por 3.2 e 3.3

$$
M_{C}=2 \beta z \mid \frac{1}{\beta}\left(-\frac{1}{2 z}+\frac{1}{|C|} \sum_{\alpha=1}^{|C|} \frac{1}{z-\zeta_{\alpha}(C)}\right) .
$$

Substituindo 3.4 em 3.1 em temos que

$$
M_{\Lambda}=-|\Lambda|^{-1} \sum_{C \subset \Lambda}|C| P_{C, \Lambda}\left(1-2 z|C|^{-1} \sum_{\alpha=1}^{|C|}\left(z-\zeta_{\alpha}(C)\right)^{-1}\right)=-p+2 z \sum_{a=1}^{N_{\Lambda}} \eta_{a}(\Lambda)\left(z-\zeta_{a}\right)^{-1}
$$

onde p é como definimos para acoplamentos com diluição nos sítios, $N_{\Lambda}$ é um inteiro finito e

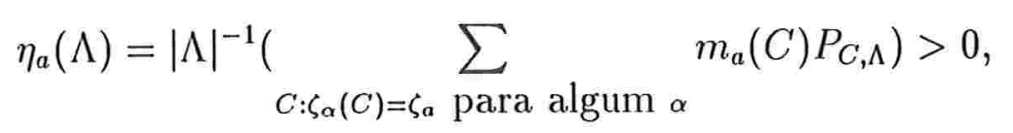

onde $m_{a}(C)$ é o número de vezes que $\zeta_{a}$ ocorre em $\left\{\zeta_{\alpha}(C)\right\}_{\alpha=1}^{|C|}$. Segue então que

$$
\sum_{a=1}^{N_{\Lambda}} \eta_{a}(\Lambda)=|\Lambda|^{-1} \sum_{C \subseteq \Lambda}\left(\sum_{\alpha=1}^{|C|} P_{C, \Lambda}\right)=\sum_{C \subseteq \Lambda}|\Lambda|^{-1}|C| P_{C, \Lambda}=p .
$$

Cálculo da magnetização: de 3.5 e 3.7 concluímos que, para $|z| \neq 1, M_{\Lambda}(z)$ é limitada uniformemente em $\Lambda$. Mais ainda, para $z \neq 1$ positivo, $M_{\Lambda}(z)$ converge para um limite, quando $\Lambda \rightarrow Z^{\nu}$, devido à existência do limite termodinâmico da energia livre ' quenched ',$f_{\Lambda}(z)$, com condições de contorno periódicas, para z positivo, e $M_{\Lambda}(z)=$ $-2 \beta z \frac{\partial f_{\Lambda}}{\partial z}$, para $z \neq 1$, positivo.

Então, pelo teorema de Vitali,

$$
M_{\Lambda}(z) \rightarrow M(z)
$$

para $\Lambda \rightarrow Z^{\nu}$, para todo $z$, com $|z| \neq 1$.

onde $\mathrm{M}$ é a magnetização no limite termodinâmico, e $f_{\Lambda}(z)$ converge, quando $\Lambda \rightarrow Z^{\nu}$, para todo $z, \operatorname{com}|z| \neq 0,1$. Seja $\beta_{0}=\beta_{0},(p=1)$ a temperatura crítica do model de Ising $\nu$ dimensional. 
Teorema 3.4.1 Para $3>3.30 . . M(z)$ nảo pode ser extendida analiticamente de $\{z:|z|>$ 1) para $\{z:|z|<1\}$. ou incersamente, de $\{z:|z|<1\}$ para $\{z:|z|>1\}$.

Demonstração: Suponha o contrário. Então cxistc algum número real $x>1$ e um $\rho>0 \cdot \operatorname{com} x-\rho<1$. tal que a série de Taylor de $M(z)$ em torno de $z=x$ tam raio de covergência $\rho$, e o disco de convergência desta série contém um arco $A, A=\{z=$ $\left.e^{i \phi}:|\phi|<\delta\right\}$, para algum $\delta>0$. Dado $\beta=\beta_{0}$ e $\delta>0$, podemos escolher $\Lambda$ grande 0 bastante de modo a conter algumas singularidades $\zeta_{a}$ de $M_{\Lambda}(z)$ ( pois para todo $\epsilon>0$ existe um cluster conexo. $\mathcal{C}$, não contornando $\Lambda$, com a propriedade que $M_{\mathcal{C}}(z)$ tenha uma singularidade em algum ponto $\zeta$, com $|\zeta-1|<\epsilon$. O resíduo, $\eta_{a}$, do pólo em $\zeta_{a}$, é dado por 3.6 2.29. Seja $\mathcal{C}_{a}$ algum aglomerado conexo de sítios em $\Lambda$ não envolvendo $\Lambda$ e tal que $M_{\mathcal{C}_{a}}(z)$ tenha uma singularidade em $\zeta_{a}$, i.e., $\zeta_{a}$ é um zero de $Z_{\mathcal{C}_{a}}$. Seja $\Omega$ o menor cubo contendo propriamente $\mathcal{C}_{a}$, e suponhamos que $\Lambda$ é a união de n disjuntos transladados, $\Omega(x)(x \in \Lambda)$, de $\Omega$. Então

$$
\eta_{a}(\Lambda) \geq|\Lambda|^{-1} \sum_{x_{1}, \ldots, x_{k} ; k=1, \ldots n} \sum_{C: C \cap \Omega\left(x_{i}\right) \supseteq \mathcal{C}_{a}\left(x_{i}\right) ; i=1, \ldots, k}^{\prime} k P_{C, \Lambda}
$$

onde $\mathcal{C}_{a}(x)$ é obtido transladando-se $\mathcal{C}_{a}$ de x. Agora

$$
\sum^{\prime} P_{C, \Lambda}=P_{\mathcal{C}_{a}}^{k}\left(1-P_{\mathcal{C}_{a}}\right)^{n-k}
$$

onde $\sum^{\prime}$ é feita sobre todas configurações $C$, tais que

$$
\begin{gathered}
C \cap \Omega\left(x_{i}\right) \supseteq \mathcal{C}_{a}\left(x_{i}\right), \text { para } i=1, \ldots, k \\
C \cap \Omega(x) \nsupseteq \mathcal{C}_{a}(x), \text { para } x \notin x_{1}, \ldots, x_{k}
\end{gathered}
$$

e $x_{1}, \ldots, x_{k}$ são dados, $\mathrm{e}$

$$
P_{\mathcal{C}_{a}}=p^{\left|\mathcal{C}_{a}\right|}(1-p)^{\left|\partial \mathcal{C}_{a}\right|}
$$

onde $\partial \mathcal{C}_{a}$ é o conjunto de sítios em $\Omega$ a distância unitária de $\mathcal{C}_{a}$ e não contidos em $\mathcal{C}_{a}$. $\mathrm{A}$ teoria do modelo de Ising em $\beta=0$ mostra-nos que 


$$
\eta_{n}(1) \geq \frac{n}{|\Lambda|} P_{\mathcal{C}_{a}}=|\Omega|^{-1} P_{\mathcal{C}_{a}}=P_{a}>0
$$

uniformemente em 1.

\section{Lema 1}

$$
\left|M_{\Lambda}\left(r \zeta_{a}\right)\right| \geq 2 \frac{\eta_{a}(\Lambda)}{r-1} \geq 2 \frac{P_{a}}{r-1},
$$

para $r>1$ uniformemente em $\Lambda$.

Demonstração: Seja $\zeta_{a}=e^{i \phi_{a}}$ e observemos a figura seguinte. Façamos $r_{a b} e^{i \phi_{a b}}=$ $r \zeta_{a}-\zeta_{b}$. Pela convexidade do disco unitário,

$$
\left|\phi_{a b}-\phi_{a}\right|<\frac{\pi}{2}, \text { para } r>1
$$

Assim, desde que $|u| \geq \Re\left(u e^{i \psi}\right)$, para $u \in \mathbb{C}$ arbitrário e $\psi \in \mathbb{R}$,

$$
\begin{gathered}
\left|M_{\Lambda}\left(r \zeta_{a}\right)\right| \geq 2 r \Re\left(\left(2 r \zeta_{a}\right)^{-1} M_{\Lambda}\left(r \zeta_{a}\right) e^{i \phi_{a}}\right) \\
=2 r\left(\frac{\eta_{a}(\Lambda)}{r-1}+\sum_{b \neq a} \frac{\eta_{b}(\Lambda)}{r_{a b}} \cos \left(\phi_{a}-\phi_{a b}\right)\right) \geq 2 r \frac{\eta_{a}(\Lambda)}{r-1}
\end{gathered}
$$

Por 3.6 e 3.9

E, por 3.8 ,

$$
2 \frac{P_{a}}{r-1}
$$

Daí $M\left(r \zeta_{a}\right)$ diverge para $r \rightarrow 1$. Isto contradiz nossa hipótese de que $M(z)$ é holomorfa em alguma vizinhança do arco A introduzido no início da demonstração do teorema. Pela simetria $\left.(h \rightarrow-h) \Leftrightarrow z \rightarrow-z^{-1}\right)$ do modelo, $M\left(z^{-1}\right)=-M(z)$, e portanto $\mathrm{M}(z)$ não tem extensão analítica de $\{z:|z|<1\}$ para $\{z:|z|>1\}$. Isto completa a demonstração do teorema. 


\section{Capítulo 4}

\section{Acoplamentos aleatórios de alcance infinito}

Neste capítulo veremos condições para as quais, no modelo de Ising com acoplamentos sendo v.a.i.i.d's de alcance infinito e decaimento exponencial, temos decaimento exponencial da funçào de dois pontos. A partir desta informação obteremos o limite termodinâmico e a unicidade da medida de Gibbs para este modelo. Neste capítulo se encontram as principais contribuições deste trabalho.

\subsection{Decaimento da função de dois pontos}

Para modelos tipo Ising com acoplamentos não-aleatórios de alcance infinito temos o seguinte resultado [Ellis]:

Teorema 4.1.1 Suponha que existam constantes positivas $c_{1}$ e $c_{2}$ tais que $J(k) \leq c_{1} e^{-c_{2}|k|}$, $\forall k \in Z^{d}$. Seja $\mathcal{J}_{0}=\sum_{k \in Z^{d}} J(k)$. Então:

(a) $<\sigma_{0} \sigma_{k}>(\beta, 0)$ decai exponencialmente pelo menos para $0<\beta<\mathcal{J}_{0}^{-1}$

(b) $\forall \beta>0$ e $h \neq 0,<\sigma_{0} ; \sigma_{k}>(\beta, h)$ decai exponencialmente. 
A partir do resultado acima para modelos nào-aleatórios é razoável nos perguntarmos se não valeria o mesmo resultado para o caso aleatório. Neste capítulo vamos demonstrar um resultado análogo para acoplamentos aleatórios de alcance infinito.

Consideremos o modelo de Ising com interaçòes aleatórias de alcance infinito dadas pela Hamiltoniana:

$$
H_{\Lambda}(\sigma)=-\sum_{<x, y>} J_{x y} \sigma_{x} \sigma_{y}
$$

$\operatorname{com} \tilde{J_{x y}}=J_{x y} \epsilon^{-\gamma|x-y|}$

onde: $J_{x y}$ são variáveis aletórias independentes identicamente distribuidas (v.a.i.i.d.'s) positivas e com média $\bar{J}=\int J_{x y} d \rho\left(J_{x y}\right)$ positiva.

$\gamma$ é uma constante real positiva

$|x-y|$ indica a distância entre os sítios $\mathrm{x}$ e $\mathrm{y}$

Definições:

$\delta_{\beta}=C \beta L^{d} e^{\gamma L} \frac{1}{d}\left(\frac{L}{2}+1\right)^{d}$

$\delta_{1}(\beta)=\sum_{x \in Z^{d}} e^{-\gamma|x|}$

$\mathcal{E}\left(J_{k_{0}} \ldots J_{k_{n}}\right)$ denota a média sobre todos os J's.

Teorema 4.1.2 No modelo de Ising definido pela Hamiltoniana acima. $\operatorname{com} \gamma>0$, se $\forall k_{0}, \ldots, k_{n}, \exists C$ independente de $n$ tal que $\mathcal{E}\left(J_{k_{0}} \ldots J_{k_{n}}\right)<C^{n+1}$ entâo existe $C_{1}>0$ e $\beta_{c}$ tal que $\delta_{1}(\beta)<1$ para $\beta<\beta_{c}$ e a seguinte desigualdade:

$$
\overline{<\sigma_{x} \sigma_{y}>} \leq C_{1} e^{-\gamma|x-y|}
$$

é satisfeita.

É importante ressaltarmos que neste enunciado $\beta_{c}$ não significa a temperatura crítica de transição de fase mas apenas um valor de temperatura abaixo do qual $\delta_{\beta}<1$. Para demonstrarmos este teorema faremos uso dos quatro lemas que vem a seguir. A estrutura é a seguinte: enunciamos e demonstramos os lemas 1 e 2. Em seguida apresentamos o enunciado do lema 3 e enunciamos e demonstramos o lema 4. A partir do lema 4 
demonstramos o lema 3. Neste ponto estamos prontos para apresentar a demonstraçào do Teorema principal.

Lema 1 Sejam $x, y \in Z^{d}$ com $|x-y| \geq L$. Sejam $\perp_{L}(x)=\left\{u \in Z^{d}:|x-u|_{\infty} \leq L\right\} \epsilon$ $\Lambda_{L}(y)=\left\{u \in Z^{d}:|y-u|_{\infty} \leq L\right\}$. Então:

$$
<\sigma_{x} \sigma_{y}=\leq \sum_{s \in \Lambda_{L}(x), t \in \Lambda_{L}(y),(s, t) \in X} \beta J_{s t} e^{-\gamma|s-t|}+\sum_{s \in \Lambda_{L}(x), t \notin \Lambda_{L}(y),(s, t) \in X} 3 . J_{s t} e^{-\gamma|s-t|}<\sigma_{t} \sigma_{y}>
$$

onde $X$ é definido por:

$X=\left\{(u, v) \in Z^{d} \times Z^{d}\right.$ tais que ou $u \in \Lambda_{L}(x) \in v \notin \Lambda_{L}(x)$ ou $u \notin \Lambda_{L}(x)$ e $\left.v \in \Lambda_{L}(x)\right\}$.

\section{Demonstração:}

Do teorema de Lieb-Simon (ver Apêndice) sabemos que

$$
<\sigma_{x} \sigma_{y}>_{\Lambda}-<\sigma_{x} \sigma_{y}>_{X} \leq \sum_{(t, s) \in X}<\sigma_{x} \sigma_{s}>_{X} \beta J_{s t}<\sigma_{t} \sigma_{y}>_{\Lambda}
$$

(Como $x \in \Lambda_{L}(x)$ e $y \notin \Lambda_{L}(x)$, pela definição do conjunto $X$ temos $<\sigma_{x} \sigma_{y}>_{X}=0$ ). Assim:

$$
<\sigma_{x} \sigma_{y}>\leq \sum_{(t, s) \in X}<\sigma_{x} \sigma_{s}>_{X} \beta J_{s t} e^{-\gamma|s-t|}<\sigma_{t} \sigma_{y}>_{\Lambda}
$$

Podemos reescrever a desigualdade 4.1 como:

$$
\begin{aligned}
\left\langle\sigma_{x} \sigma_{y}\right\rangle \leq \sum_{s \in \Lambda_{L}(x), t \in \Lambda_{L}(y),(s, t) \in X}<\sigma_{x} \sigma_{s}>_{X} \beta J_{s t} e^{-\gamma|s-t|}<\sigma_{t} \sigma_{y}>_{\Lambda}+ \\
\sum_{s \in \Lambda_{L}(x), t \notin \Lambda_{L}(y),(s, t) \in X}<\sigma_{x} \sigma_{s}>_{X} \beta J_{s t} e^{-\gamma|s-t|}<\sigma_{t} \sigma_{y}>_{\Lambda}
\end{aligned}
$$

Utilizando as limitações triviais $\left\langle\sigma_{x} \sigma_{s}\right\rangle_{x} \leq 1 \mathrm{e}<\sigma_{t} \sigma_{y}>_{1} \leq 1$ na desigualdade anterior, obtemos: 


$$
\left.\left.<\sigma_{x} \sigma_{y}\right\rangle \leq \sum_{s \in \Lambda_{L}(x), t \in \Lambda_{L}(y) .(s, t) \in X} 3 J_{s t} \epsilon^{-\gamma|s-t|}+\sum_{s \in \Lambda_{L}(x) . t \notin \Lambda_{L}(y) .(s, t) \in X} 3 J_{s t} \epsilon^{-\gamma|s-t|}<\sigma_{t} \sigma_{y}\right\rangle
$$

E concluímos a demonstração do lema.

Enuciaremos e demonstraremos agora um lema indutivo:

Lema 2 Suponha que:

$$
\begin{aligned}
& <\sigma_{x} \sigma_{y}>\leq \sum_{s_{0} \in \Lambda_{L}(x), t_{0} \in \Lambda_{L}(y)} \beta J_{s_{0} t_{0}} e^{-\gamma\left|s_{0}-t_{0}\right|}+ \\
& \sum_{s_{0} \in \Lambda_{L}(x), t_{0} \notin \Lambda_{L}(y)} \sum_{s_{1} \in \Lambda_{L}\left(t_{0}\right), t_{1} \in \Lambda_{L}(y)} \beta J_{s_{0} t_{0}} e^{-\gamma\left|s_{0}-t_{0}\right|} \beta J_{s_{1} t_{1}} e^{-\gamma\left|s_{1}-t_{1}\right|}+\ldots+ \\
& \sum_{s_{0} \in \Lambda_{L}(x), t_{0} \notin \Lambda_{L}(y)} \sum_{s_{1} \in \Lambda_{L}\left(t_{0}\right), t_{1} \notin \Lambda_{L}(y)} \ldots \sum_{s_{n} \in \Lambda_{L}\left(t_{n-1}\right), t_{n} \in \Lambda_{L}(y)} \beta \sum_{s_{s_{0} t_{0}} e^{-\gamma\left|s_{0}-t_{0}\right|} \ldots \beta J_{s_{n} t_{n}} e^{-\gamma\left|s_{n}-t_{n}\right|}} \sum_{s_{1} \in \Lambda_{L}\left(t_{0}\right), t_{1} \notin \Lambda_{L}(y)} \ldots \sum_{s_{n} \in \Lambda_{L}\left(t_{n-1}\right), t_{n} \notin \Lambda_{L}(y)} \beta J_{s_{0} t_{0}} e^{-\gamma\left|s_{0}-t_{0}\right|} \ldots \beta J_{s_{n} t_{n}} e^{-\gamma\left|s_{n}-t_{n}\right|}<\sigma_{i_{n}} \sigma_{y}>
\end{aligned}
$$

onde $\left|s_{j}-t_{j}\right| \leq \frac{L}{2}, \forall 1 \leq j \leq n$

então:

$$
\begin{aligned}
& <\sigma_{x} \sigma_{y}>\leq \sum_{s_{0} \in \Lambda_{L}(x), t_{0} \in \Lambda_{L}(y)} \beta J_{s_{0} t_{0}} e^{-\gamma\left|s_{0}-t_{0}\right|}+ \\
& \sum_{s_{0} \in \Lambda_{L}(x), t_{0} \notin \Lambda_{L}(y)} \sum_{s_{1} \in \Lambda_{L}\left(t_{0}\right), t_{1} \in \Lambda_{L}(y)} \beta J_{s_{0} t_{0}} e^{-\gamma\left|s_{0}-t_{0}\right|} \beta J_{s_{1} t_{1}} e^{-\gamma\left|s_{1}-t_{1}\right|}+\ldots+ \\
& \sum_{s_{0} \in \Lambda_{L}(x), t_{0} \notin \Lambda_{L}(y)} \cdots \sum_{s_{n+1} \in \Lambda_{L}\left(t_{n}\right), t_{n+1} \in \Lambda_{L}(y)} \beta J_{s_{0} t_{0}} e^{-\gamma\left|s_{0}-t_{0}\right|} \ldots \beta J_{s_{n+1} t_{n+1}} e^{-\gamma\left|s_{n+1}-t_{n+1}\right|} \cdot 1+ \\
& \sum_{s_{0} \in \Lambda_{L}(x), t_{0} \notin \Lambda_{L}(y)} \ldots \sum_{s_{n+1} \in \Lambda_{L}\left(t_{n}\right), t_{n+1} \notin \Lambda_{L}(y)} \beta J_{s_{0} t_{0}} e^{-\gamma\left|s_{0}-t_{0}\right|} \ldots \beta J_{s_{n+1} t_{n+1}} e^{-\gamma\left|s_{n+1}-t_{n+1}\right|}<\sigma_{t_{n+1}} \sigma_{y}>
\end{aligned}
$$

onde $\left|s_{j}-t_{j}\right| \leq \frac{L}{2}, \forall 1 \leq j \leq n+1$

\section{Demonstração:}

A prova consiste em aplicarmos o lema $1 \mathrm{em}\left\langle\sigma_{t_{n}} \sigma_{y}\right\rangle$ e desenvolvermos o produto. 
Agora temos a demonstraçào do lema 3:

\section{Demonstração:}

Pelo fato de $\mathcal{E}\left(J_{k_{0}} \ldots J_{k_{n}}\right)<C^{n+1}$ temos a seguinto desigualdade:

$$
\begin{aligned}
& \sum_{s_{0} \in \Lambda_{L}(x), t_{0} \in \Lambda_{L}\left(t_{1}\right)} \ldots \sum_{s_{n} \in \Lambda_{L}\left(t_{n-1}\right), t_{n} \in \Lambda_{L}(y)} \beta J_{s_{0} t_{0}} \epsilon^{-\gamma\left|s_{0}-t_{0}\right|} \ldots \beta J_{s_{n} t_{n}} \epsilon^{-\gamma\left|s_{n}-t_{n}\right|} \leq \\
& \leq(C \beta)^{n+1} \sum_{s_{0} \in \Lambda_{L}(x), t_{0} \in \Lambda_{L}\left(t_{1}\right)} \ldots \sum_{s_{n} \in \Lambda_{L}\left(t_{n-1}\right), t_{n} \in \Lambda_{L}(y)} e^{-\gamma\left|s_{0}-t_{0}\right|} \ldots e^{-\gamma\left|s_{n}-t_{n}\right|}
\end{aligned}
$$

Como $s_{0} \in \Lambda_{L}(x)$, temos que $\left|s_{0}-t_{0}\right| \geq\left|x-t_{0}\right|-L$ e portanto $e^{-\gamma\left|s_{0}-t_{0}\right|} \leq e^{-\gamma\left|x-t_{0}\right|+\gamma L}$.

Temos ainda que (para $j>1$ ): $s_{j} \in \Lambda_{L}\left(t_{j-1}\right) \Rightarrow\left|s_{j}-t_{j}\right| \geq\left|t_{j-1}-t_{j}\right|-L$. E portanto $e^{-\gamma|s,-t,|} \leq \epsilon^{-\gamma\left|t_{3}-1-t_{3}\right|+\gamma L}$.

E como $\sum_{s_{j} \in \Lambda_{L}\left(t_{j-1}\right)} 1=L^{d}$, podemos majorar 4.6 por:

$$
\left(C \beta L^{d} e^{\gamma L}\right)^{n+1} \sum_{t_{0} \in Z^{d}} \sum_{t_{1} \in Z^{d}} \ldots \sum_{t_{n} \in Z^{d}} e^{-\gamma\left|x-t_{0}\right|-\gamma\left|t_{0}-t_{1}\right|-\ldots-\gamma\left|t_{n}-y\right|}
$$

onde $\left|x-t_{0}\right| \leq \frac{L}{2},\left|t_{n}-t_{n+1}\right| \leq \frac{L}{2}, \forall n \geq 0,\left|t_{n}-y\right| \leq \frac{L}{2}$.

Aplicando o lema 4 à desigualdade anterior, podemos majorá-la por:

$\left(C \beta L^{d} e^{\gamma L}\left[\frac{1}{d}\left(\frac{L}{2}+1\right)^{d}\right]\right)^{n+1} e^{-\gamma|x-y|}$

e concluímos o lema.

Neste ponto estamos prontos para demonstrar o Teorema principal:

\section{Demonstração:}

Do Lema 2 e do Lema 3 temos que:

$$
\overline{<\sigma_{x} \sigma_{y}>} \leq \sum_{j=1}^{n-1} \delta_{\beta}^{j} e^{\gamma|x-y|}+\delta_{\beta}^{n} \sum_{t_{0} \notin \Lambda_{L}(y)} \cdots \sum_{t_{n} \notin \Lambda_{L}(y)} e^{-\gamma\left|x-t_{0}\right|-\ldots-\gamma\left|t_{n-1}-t_{n}\right|} .1
$$

Onde, na última somatória da segunda parcela da desigualdade anterior, fixado $t_{n-1}$, somamos sobre todos os valores de $t_{n}$, obtendo assim:

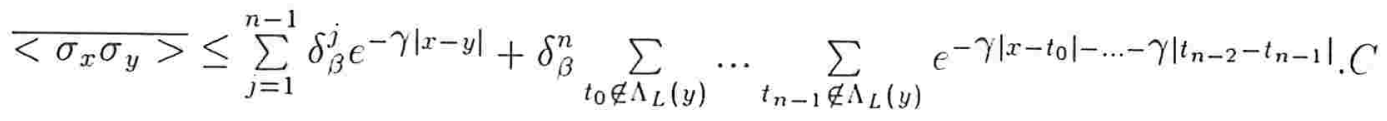


Como $\sum_{t_{n} \notin \Lambda_{L}(y)} \epsilon^{-\gamma\left|t_{n-1}-t_{n}\right|} \leq \sum_{t \in Z^{d}} t^{-\gamma|t|} \equiv C^{\prime}$

Repetindo este mesmo argumento sucessivamente (fixando $t_{n-2}$ e somando sobre $t_{n-1}$ e assim por diante) obtemos a desigualdade:

$\overline{<\sigma_{x} \sigma_{y}>} \leq \sum_{j=1}^{n-1} \delta_{\beta}^{j} c^{-\gamma|x-y|}+\left(\delta_{3} C\right)^{n}$

Seja $\delta_{1}(3)=\delta_{3} C$.

Escolhemos 3 tal que $\delta_{1}(\beta)<1$.

E então:

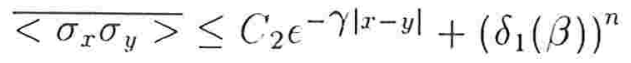

$\operatorname{com} C_{2}=\sum_{j=1}^{\infty} \delta_{\beta}^{j}=\frac{\delta_{\beta}}{1-\delta_{\beta}}$

Como n é arbitrário, escolhemos n tal que $n \ln \left(\delta_{1}(\beta)\right)=-\gamma|x-y|$ e portanto:

$\overline{\left\langle\sigma_{x} \sigma_{y}\right\rangle} \leq\left(\frac{\delta_{\beta}}{1-\delta_{\beta}}+1\right) e^{-\gamma|x-y|}=\frac{1}{1-\delta_{\beta}} \epsilon^{-\gamma|x-y|}=C_{1} \epsilon^{-\gamma|x-y|}$

onde $C_{1}$ é uma constante positiva.

Donde concluímos a demonstração

\subsection{Independência da Magnetização em relação às condições de Fronteira}

A importância do teorema anterior está em que podemos utilizá-lo para demonstrar o limite termodinâmico quando tomamos caixas arbitrariamente grandes (tendendo ao espaço todo), sem que haja dependência das condições de fronteira. Ou seja, que temos um único estado de Gibbs para altas temperaturas. A técnica que será utilizada para a demonstração deste fato é a de duplicaçào de variáveis.

Teorema 4.2.1 Nas mesmas hipóteses do teorema anterior, existe $\beta_{c}$ tal que $\forall 0<\beta<\beta_{c}$ existem constantes $0<C(\beta)<\infty$ tal que, para quaisquer condiçôes de contorno $\chi_{\Lambda}$ temos:

(a) $\overline{\left.<\sigma_{x} ; \sigma_{y}\right\rangle_{\Lambda}^{\Lambda}} \leq C e^{-\gamma|x-y|}$

(b) existe o limite termodinâmico e é independente das condiçôes de fronteira $\chi_{\Lambda}$ dadas para cada volume $\Lambda$ finito: 


$$
\overline{\left\langle\sigma_{x}\right\rangle}=\lim _{.1 \rightarrow Z^{d}} \overline{\left\langle\sigma_{x}>{ }_{.1}\right.}
$$

Demonstração: (a) I'sando o truque de duplicação de variáveis consideremos dois conjuntos de variáveis dos sítios:

$$
\sigma^{1}: \Lambda \rightarrow R \sigma^{2}: \Lambda \rightarrow R
$$

com Hamiltoniana $\check{H}_{\Lambda}$ dada por

$$
\tilde{H}_{\Lambda}\left(\sigma^{1}, \sigma^{2}\right)=H_{\Lambda}\left(\sigma^{1}\right)+H_{\Lambda}\left(\sigma^{2}\right)
$$

e as funções dois spins $\tilde{F}$ e $\tilde{G}$ dadas por

$$
\tilde{F}_{\Lambda}\left(\sigma^{1}, \sigma^{2}\right)=F_{\Lambda}\left(\sigma^{1}\right)+F_{\Lambda}\left(\sigma^{2}\right)
$$

e

$$
\tilde{G}_{\Lambda}\left(\sigma^{1}, \sigma^{2}\right)=G_{\Lambda}\left(\sigma^{1}\right)+G_{\Lambda}\left(\sigma^{2}\right)
$$

com valores esperados dos spins dados por:

$$
<<\tilde{F}>>_{\Lambda}^{\chi_{\Lambda}}=\frac{\iint \tilde{F}_{\Lambda}\left(\sigma^{1}, \sigma^{2}\right) e^{-\beta \tilde{H}_{\Lambda}\left(\sigma^{1}, \sigma^{2}\right)} \prod_{x \in \Lambda} d \mu_{0}\left(\sigma_{x}^{1}\right) d \mu_{0}\left(\sigma_{x}^{2}\right)}{\tilde{Z}_{\Lambda}^{\lambda}}
$$

onde $\tilde{Z}_{\Lambda}^{\chi}$ é a função de partição do sistema duplicado:

$$
\tilde{Z}_{\Lambda}^{\lambda}=\int e^{-\beta \tilde{H}_{\Lambda}\left(\sigma^{1}, \sigma^{2}\right)} \prod_{x \in \Lambda} d \mu_{0}\left(\sigma_{x}^{1}\right) d \mu_{0}\left(\sigma_{x}^{2}\right)
$$

Observando que com as novas variáveis

$$
\begin{aligned}
& \eta_{x}=\frac{1}{\sqrt{2}}\left(\sigma_{x}^{1}+\sigma_{x}^{2}\right) \\
& \zeta_{x}=\frac{1}{\sqrt{2}}\left(\sigma_{x}^{1}-\sigma_{x}^{2}\right)
\end{aligned}
$$

a medida $d \mu_{0}(\eta, \zeta)=d \mu_{0}\left(\frac{\eta+\zeta}{\sqrt{2}}\right) d \mu_{0}\left(\frac{\eta-\zeta}{\sqrt{2}}\right)$ é invariante em relação às transformaçòes :

$$
\begin{gathered}
(\eta, \zeta) \rightarrow(-\eta, \zeta) \\
(\eta, \zeta) \rightarrow(-\eta,-\zeta)
\end{gathered}
$$

e que (com acoplamentos positivos ) $\tilde{H}_{\Lambda}\left(\sigma^{1}, \sigma^{2}\right)=H_{\Lambda}(\eta, \zeta)$ teremos: 


$$
<\sigma_{x}: \sigma_{y}>_{.1}^{1.1}=<<\zeta_{x} \zeta_{y}>>_{.1}^{1.1}=<\dot{\zeta}_{x} \dot{\zeta}_{y}>_{.1}
$$

Entào:

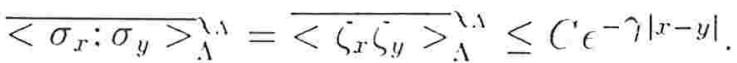

Sendo que a última desigualdade sai do teorema anterior. O que conclui a parte (a) do teorema.

Definição: dizemos que fixamos uma condiçào de fronteira $\backslash \wedge$ se $\forall y \in \partial . \Lambda$ temos $\left\{\sigma_{y}\right\}=\chi_{\Lambda}$.

Para a parte (b) temos, do teorema fundamental do Cálculo que:

$<\sigma_{0}>_{\Lambda}^{\Lambda}-<\sigma_{0}>_{\Lambda}=\int_{0}^{1} d s \frac{d}{d s}<\sigma_{0}>^{s \backslash \Lambda}$

E, efetuando a derivação na equação anterior obtemos:

$\int_{0}^{1} d s \frac{d}{d s}<\sigma_{0}>^{s \chi \Lambda}=\sum_{x \in \Lambda, y \in \Lambda^{c}} \tilde{J}_{x y}<\sigma_{0} ; \sigma_{x} \sigma_{y}>^{\wedge \Lambda}$

Onde $\sigma_{y}$ é fixado a partir da condição de fronteira $\chi_{\Lambda}$.

A desigualdade anterior é majoràda por:

$$
\sum_{x \in \Lambda, y \in \Lambda^{c}} \tilde{J_{x y}}<\sigma_{0} ; \sigma_{x}>^{\chi_{\Lambda}}
$$

Pois esta majoração vale tanto para $\sigma_{y}=+1$ quanto para $\sigma_{y}=-1$.

Aplicando a técnica de duplicação de variáveis, temos a igualdade:

$$
\sum_{x \in \Lambda, y \in \Lambda^{c}} \tilde{J_{x y}}<\sigma_{0} ; \sigma_{x}>\wedge=\sum_{x \in \Lambda, y \in \Lambda^{c}} \tilde{J_{x y}}<<\zeta_{0} \zeta_{x}>>
$$

Usando agora que $\tilde{J_{x y}} \leq J_{x y} e^{-\gamma|x-y|}$ e que $\left\langle\left\langle\zeta_{0} \zeta_{x}>>\leq e^{-\gamma|x|}\right.\right.$, a igualdade anterior é majorada por:

$$
C \sum_{x \in \Lambda, y \in \Lambda^{c}} e^{-\gamma|x-y|} e^{-\gamma|x|}
$$

Aplicando o lema 4 deste capítulo obtemos:

$$
\text { C. } \sum_{x \in \Lambda, y \in \Lambda^{c}} e^{-\gamma|x-y|} e^{-\gamma|x|} \leq C \Lambda^{d} \sum_{y \in \Lambda^{c}} e^{-\gamma|y|}
$$

Portanto: 
$\left|\overline{\left.\left\langle\sigma_{0}\right\rangle_{.1}^{1.1}-<\sigma_{0}\right\rangle_{.1}}\right| \leq C^{\prime} . I^{l} \sum_{y \in \Lambda^{c}} \epsilon^{-\gamma|y|} \rightarrow 0$

O que implica que $\left.\lim _{. \rightarrow Z^{a}}<\sigma_{0}>\right\rangle_{.1}^{.1}=\lim _{\Lambda \rightarrow Z^{d}}<\sigma_{0}>_{1}$

Este limite implica que nào há dependência em relaçiò às condiçòes de fronteira. quando estamos em regime de altas temperaturas. Ou seja. temos a existência do limite termodinâmico e há um único estado de Gibbs. 


\section{Apêndice A}

\section{Desigualdades de Correlação}

\section{A.1 Primeira Desigualdade de Griffiths}

Teorema A.1.1 Consideremos um subconjunto $A \subset \Lambda \subset Z^{d}$ e $\sigma_{A} \equiv \prod_{x \in A} \sigma_{x}$. Então:

$$
<\sigma_{A}>\geq 0
$$

Demonstração: Temos que $<\sigma_{A}>_{\Lambda}=\frac{1}{Z_{\Lambda}} \int_{\Omega_{\Lambda}} \sigma_{A} e^{-H_{\Lambda}(\{\sigma\})}=\frac{1}{Z_{\Lambda}} \int_{\Omega_{\Lambda}} \sigma_{A} e^{\sum_{x y} J_{x y} \sigma_{x} \sigma_{y}} \prod_{x \in \Lambda}$. Expandindo $e^{\sum_{x y \in \Lambda} J_{x y} \sigma_{x} \sigma_{y}}$ em série de Taylor e trocando a ordem da soma com a ordem de integração ficamos com:

$$
<\sigma_{A}>_{\Lambda}=\frac{1}{Z_{\Lambda}} \sum_{k=0}^{\infty} \frac{1}{k} \int\left(\sum_{x y \in \Lambda} J_{x} y \sigma_{x} \sigma_{y}\right)^{k} \sigma_{A} \prod_{x \in \Lambda} d \mu_{o}\left(\sigma_{x}\right) .
$$

Para demonstrarmos o teorema basta mostrarmos que $\int\left(\sum_{x y \in \Lambda}\right)^{k} \sigma_{A} \prod_{x \in \Lambda} d \mu_{o}\left(\sigma_{x}\right) \geq$ $0, \forall k \geq 0$ pois $Z_{\Lambda}$ é sempre maior ou igual a zero.

A partir do desenvolvimento de $\left(\sum J_{x y} \sigma_{x} \sigma_{y}\right)^{k}$ obtemos termos do tipo $\prod_{x \in B} \sigma_{x} \prod_{x \in A} \sigma_{x}$. Portanto, devemos mostrar que $\int \prod_{x \in C} \sigma_{x} \prod_{x \in \Lambda} d \mu_{o}\left(\sigma_{x}\right) \geq 0$. 


\section{A.2 Segunda Desigualdade de Griffiths}

Teorema A.2.1 Consideremos os subconjuntos $A \subset A \subset Z^{d} \in B \subset . \subset Z^{d} \in \sigma_{A} \equiv \prod_{x \in A}$ $\epsilon \sigma_{B} \equiv \prod_{x \in B}$. Então:

$$
<\sigma_{A}: \sigma_{B}>=<\sigma_{A} \sigma_{B}>-<\sigma_{A}><\sigma_{B}>\geq 0
$$

Demonstração: para demonstrar esta desigualdade utilizamos a técnica de Duplicaçào do Modelo. Consideremos duas famílias de funções:

$$
\begin{gathered}
\sigma: \Lambda \rightarrow R \\
\sigma: \Lambda \rightarrow R
\end{gathered}
$$

uma função Energia dada por:

$$
\tilde{H}\left(\sigma, \sigma^{\prime}=H(\sigma)+H\left(\sigma^{\prime}\right)\right.
$$

e valores esperados definidos por :

$$
<<F\left(\sigma, \sigma^{\prime}\right)>>\equiv \iint F\left(\sigma, \sigma^{\prime}\right) e^{-\beta \hat{H}\left(\sigma, \sigma^{\prime}\right)} \prod_{x \in \Lambda} d \mu_{o}\left(\sigma_{x}\right) d \mu_{o}\left(\sigma_{x}^{\prime}\right)
$$

Definindo as variáveis :

$$
\begin{aligned}
& \eta_{x}=\frac{1}{\sqrt{2}}\left(\sigma_{x}+\sigma_{x}^{\prime}\right) \\
& \zeta_{x}=\frac{1}{\sqrt{2}}\left(\sigma_{x}-\sigma_{x}^{\prime}\right)
\end{aligned}
$$




\section{A.3 Aplicações de G I e G II}

C'omo exemplo de aplicaçào das desigualdades de Ciriffiths veremos a demonstraçào da existência do limite termodinâmico para o modelo de Ising. C'onsideremos o modelo de Ising com hamiltoniana dada por

$$
H_{\Lambda}(\sigma)=-\sum_{(x, y) \in \Lambda,|x-y|=1} \sigma_{x} J_{x y} \sigma_{y}
$$

onde

$$
J_{x y}=\left\{\begin{array}{l}
J_{y x}=J>0 \text { se }|x-y|=1 \\
\text { 0caso contrário }
\end{array}\right.
$$

e $\sigma_{x} \in-1,1$ para $x \in \Lambda \subset Z^{d}$.

E condições de contorno livres. Consideremos ainda $\Lambda^{\prime}$ uma outra caixa contida em $Z^{d}$ tal que $\Lambda^{\prime} \supset \Lambda$. Temos então o seguinte

Teorema A.3.1 Se F'é um polinômio com coeficientes positivos e temos acoplamentos como os definidos anteriormentc. Então:

$$
\Lambda \subset \Lambda^{\prime} \rightarrow<F>_{\Lambda} \leq<F>_{\Lambda^{\prime}}
$$

Demonstração: Definamos a seguinte função

$$
<F>_{t}=\frac{\int F g\left(\sigma_{x}^{2}\right) \prod_{y \in \Lambda^{\prime} \backslash \Lambda} g\left(\sigma_{y}^{2}\right) e^{-t_{y} \sigma_{y}^{2}} \prod_{x \in \Lambda} e^{-\frac{1}{2} \beta H_{\Lambda}(\sigma)} d \sigma_{x}}{\int e^{-\frac{1}{2} \beta H_{\Lambda}(\sigma)} \prod_{x \in \Lambda} g\left(\sigma_{x}^{2}\right) e^{-t_{y} \sigma_{y}^{2}} d \sigma_{x}}
$$

Assim temos: $\langle F\rangle_{t=0}=\langle F\rangle_{\Lambda^{\prime}}$ e $\langle F\rangle_{t=\infty}=\langle F\rangle_{\Lambda}$. Portanto basta provarmos que $\frac{\partial\left(\langle F\rangle_{t}\right)}{\partial t_{y}} \leq 0, \forall y \in \Lambda^{\prime} \backslash \Lambda$

Fazendo a derivada obtemos:

$\frac{\left.\partial(<F\rangle_{t}\right)}{\tilde{\partial} t_{y}}=-<F \sigma_{y}^{2}>+<F><\sigma_{y}^{2}>\leq 0$ ( pela segunda desigualdade de Gifriths). Esta última desigualdade implica na monotonicidade dos valores esperados quando o volume decresce. A partir da limitação uniforme nos valores esperados temos a existência do limite termodinâmico $\left(\Lambda \rightarrow Z^{d}\right.$ :

$$
\lim _{\Lambda \rightarrow Z^{d}}<F>_{\Lambda}=<F>_{Z^{d}}
$$




\section{A.4 Desigualdade FKG}

A desigualdade Fhi i tem a mesma tese que a Segunda desigualdade de Griffiths. só que sob hipóteses diferentes. É importante ainda frisarmos que este desigualdarle também é válida sob medidas mais gerais que a medida de Gibbs. Neste sentido, por exemplo. consultar [Ellis].

Definimos uma ordem em $R^{n}$ através da relação :

$$
\xi=\left(\xi_{1}, \xi_{2}, \ldots, \xi_{n}\right) \leq \lambda=\left(\lambda_{1}, \chi_{2}, \ldots, \backslash_{n}\right) \Longleftrightarrow \xi_{i} \leq \backslash_{i}, \forall i .
$$

Uma função $F(\xi)$ é chamada monótona se é monótona com respeito a esta ordem.

Teorema A.4.1 Sejam Fe Ci funçòes monótonas crescentes em l e seja a esperança definida $\langle\cdot\rangle$ definida por : $\langle F(\lambda)\rangle=\int F e^{\frac{1}{2} \beta H(\xi)} \prod_{i \in \Lambda} d \mu_{i}$

onde $H(\xi)=-\sum_{i, j \in \Lambda}\left(\xi_{i}-\xi_{j}\right)^{2}$ ed $\mu_{i}=\frac{\epsilon^{-P\left(\xi_{1}\right)} d \xi_{1}}{\int e^{-P} d \xi_{2}}$

Então $\langle F G\rangle \geq\langle F\rangle\langle G\rangle$

\section{Demonstração}

Com as variáveis duplicadas $\xi$ e $\lambda$, devemos mostrar que

$$
0 \leq<[F(\xi)-F(\chi)][G(\xi)-G(\chi)]>.
$$

Seja $n=|\Lambda|$ o número de sítios em $\Lambda$. Para $n=1, F(\xi)-F(\lambda)$ e $G(\xi)-G(\lambda)$ têm o mesmo sinal pela monotonicidade, e temos a tese neste caso.

Suponhamos agora que o teorema tenha sido provado para n - 1 sítios e escrevamos $\xi=\left(\xi, \xi_{n}\right)$, onde $\xi=\left(\xi_{1}, \xi_{2}, \ldots, \xi_{n-1}\right)$ e analogamente para $\chi$.

Escrevendo $\left(^{*}\right)$ como uma integral iterada, mostremos que a integral em $d \xi d \backslash$ é nào negativa para cada valor de $\backslash_{n}, \xi_{n}$.

Explicitamente,

$$
Z(a)=<\delta\left(\xi_{n}-a\right)>=\int \prod_{j \neq n} d \xi_{j} \int d \xi_{n} F(\xi) \epsilon^{-3 H(\xi)} e^{-P(\xi)}
$$




$$
=\left.\left.\int \prod_{i \neq n} d \xi_{j} l \cdot(\xi)\right|_{\xi_{n}=\alpha} e^{-\beta / l(\xi)}\right|_{\xi_{n}=\alpha} \prod_{j} e^{-l^{\prime}\left(\xi_{\jmath}\right)} e^{-P(v)}
$$

$\mathrm{e}$

$$
<F>_{\xi_{n}=\alpha}=Z(\alpha)^{-1}<\delta\left(\xi_{n}-\alpha\right) F(\xi)>=\frac{\sum_{\xi: \xi_{n}=\alpha} F(\xi) e^{-\frac{1}{2} \beta H(\xi)}}{\sum_{\xi: \xi_{n}=\alpha} e^{-\frac{1}{2} \beta H(\xi)}}
$$

Vamos denotar a esperança do sistema com variáveis duplicadas da seguinte maneira

$$
<\cdot>_{\xi_{n}=\alpha, \chi n=\gamma} \equiv<\cdot>_{\alpha, \gamma}
$$

Temos a identidade :

$$
\begin{aligned}
& <[F(\xi)-F(\chi)][G(\xi)-G(\gamma)]>_{\alpha, \gamma}=<F G>_{\alpha}+<F G>_{\gamma}-<F>_{\alpha}<G>_{\gamma}-<F>_{\gamma}<G>_{\alpha} \\
& =\left[<F G>_{\alpha}-<F>_{\alpha}<G>_{\alpha}\right]+\left[<F G>_{\gamma}-<F>_{\gamma}<G>_{\gamma}\right]+\left[<F>_{\alpha}-<G>_{\gamma}\right]\left[<G>_{\alpha}-\right. \\
\left.<G>_{\gamma}\right] \quad(* * *) &
\end{aligned}
$$

Devemos mostrar que esta última identidade é não-negativa. Os dois primeiros termos sào não-negativos pela hipótese de indução e assim, para a demonstração do teorema estará completa se mostrarmos que os fatores do terceiro termo tem o mesmo sinal. Faremos isto usando a dependência de $\mathrm{Z} \operatorname{em} \alpha$ dada por ${ }^{* *}$ ) e a definição do valor esperado yzyzyzyzy.

$$
\begin{aligned}
& \frac{d}{d \alpha}<F>_{\alpha}=\frac{d}{d \alpha}\left(\frac{\sum_{\xi=\xi_{n}=\alpha} F(\xi) e^{-\frac{1}{2} \beta H(\xi)}}{\sum_{\xi: \xi_{n}=\alpha} e^{-\frac{1}{2} \beta H(\xi)}}\right) \\
& =\left\langle\frac{d F}{d \xi_{x}}\right\rangle_{\alpha}+\beta \sum_{l}\left\{\left\langle\left(\xi_{l}-\alpha\right) F(\xi)>_{\alpha}-<\left(\xi_{l}-\alpha\right)>_{\alpha}\left\langle F>_{\alpha}\right\}\right.\right.
\end{aligned}
$$

onde a soma $\mathrm{em} l$ se estende sobre os sítios que sào vizinhos próximos do n-ésimo sítio. Observe que os termos proporcionais a $P^{\prime}\left(\xi_{n}\right)$ se cancelam. Como a função $\xi \rightarrow \alpha-\xi_{l}$ é monótona, a hipótese de indução se aplica novamente e disto segue que $\langle F\rangle_{\alpha}$ é monotona. crescente em $\alpha$. O mesmo é válido para $\langle G\rangle_{\alpha}$ e assim o terceiro termo de ( $\left.{ }^{* * *}\right)$ é nãonegativo. 


\section{A.5 Teorema de Lieb-Simon}

Teorema A.5.1 (onsideremos um conjunto $X$ de pares (u.v) de pontos da rede 1 . onde os pares $(u, v) \in(v, u)$ são considerados distintos e $\mathrm{X}$ é um conjunto tal que se $(u, v) \in X^{\prime}$ então $(v, u) \in X^{\prime}$. Definimos $\left\langle()>\right.$. colocando $J_{u v}=0$ para todo uv $\in X$ na definiçào de $<()>._{\Lambda}$. Então para todo $x, !^{\prime} \in \Lambda$, temos que:

$$
0 \leq<\sigma_{x} \sigma_{y}>_{\Lambda}-<\sigma_{x} \sigma_{y}>_{X} \leq \sum_{u v \in X}<\sigma_{x} \sigma_{u}>_{X} J_{u v}<\sigma_{v} \sigma_{y}>_{\Lambda}
$$

Este teorema foi utilizado em diversos pontos deste trabalho. Para a demonstração dele, usaremos o teorema das desigualdades gaussianas:

Teorema A.5.2 Sc Fé um polinômio com coeficientes positivos, entĩo:

$$
<\sigma_{x} F(\sigma)>\leq \sum_{y}<\sigma_{x} \sigma_{y}><\frac{\partial F(\sigma)}{\partial \sigma}>
$$

\section{Demonstração:}

Da definição de $\langle(.)\rangle_{X}$ temos que:

$$
<\sigma_{x} \sigma_{y}>=\frac{<\sigma_{x} \sigma_{y} I>_{X}}{<I>_{X}}
$$

onde

$$
I=e^{\sum_{u v \in X} \sigma_{u} \sigma_{v}}
$$

I é o limite de polinômios com coeficientes positivos e este limite pode ser trocado com as integrações de forma a podermos aplicar os resultados do Teorema das Desigualdades Gaussianas para $\langle(.)\rangle_{x} \operatorname{com} F(\sigma)=\sigma_{y} I$ e obter:

$$
<\sigma_{x} \sigma_{y} I>_{X} \leq<\sigma_{x} \sigma_{y}>_{X}<I>_{X}+<\sigma_{x} \sigma_{u}>_{X} J_{u v}<\sigma_{\imath} \sigma_{y} I>_{X}
$$


Dividindo por $\langle I\rangle_{x}$ obtemos:

$$
<\sigma_{x} \sigma_{y}>\leq<\sigma_{x} \sigma_{y}>_{X}+\sum_{u v \in X}<\sigma_{x} \sigma_{w}>_{X} J_{w z}<\sigma_{z} \sigma_{y}>
$$




\section{Bibliografia}

[1] D. Brydges.

Field Theories and Symanzik's Polymer Representation

Brasov Lectures, 1981.

[2] H. von Dreifus.

Annales Inst. Henri Poincaré

vol. 55, no. 2, 1991, p. 657.

[3] H. von Dreifus.

Mecânica Estatistica de Modelos Ferromagnéticos

19o. Colóquio Brasileiro de Matemática (IMPA), 1993

[4] Ellis, R.

Entropy, Large Deviations and Statistical Mechanics

Springer-Verlag

[5] Fortuin,Kasteleyn e Ginibre

Correletion inequalities on some partially ordered sets

Commun. Math. Phys. 22, p. 89-113.

[6] J. Fröhlich.

Mathematical Aspects of the physics of disordered systems

Les Houches, Session XLIII, 1984, p. 725.

[i] Georgii, H.O.

Gibbs Measures and Phase Transitions

Walter de Gryter, 1988. 
[8] J. Glimm, A. Jaffe.

Quantum Physics

Springer-Verlag, 1981.

[9] Graham

Applications of the FKG inequality and its relatives

in: Mathematical Progamming - Bonn 1982, p. 116-131. Springer - NY

Edited by A. Bachem, M. Grotschel, B. Korte.

[10] McCoy e Wu.

The Two-dimensional Ising Model

Harvard University Press - Cambridge.

[11] Robert B. Griffiths.

Nonanalytic behavior above the critical point in a random ising ferromagnet

Physical Reviem Letters, vol. 23, no. 1, Julho/1969.

[12] Thompson, Colin

Mathematical Statistical Mechanics

Princeton University Press. 